\title{
Dibenzoylbenzodipyrroles: Key Precursors for the Synthesis of Fused Meso-Aryl Sapphyrins
}

\author{
Prosenjit Isar and Mangalampalli Ravikanth* \\ Department of Chemistry, Indian Institute of Technology Bombay, Powai, Mumbai 400076, \\ India, E-mail: ravikanth@chem.iitb.ac.in
}

\begin{tabular}{|c|c|c|}
\hline Figure S1 & ${ }^{1} \mathrm{H}$ NMR of compound 3a recorded in $\mathrm{CDCl}_{3}$ & $\mathbf{S 2}$ \\
\hline Figure S2 & HRMS spectrum of compound $\mathbf{3 a}$ & S3 \\
\hline Figure S3 & ${ }^{13} \mathrm{C}$ NMR of compound 3arecorded in $\mathrm{CDCl}_{3}$ & $\mathbf{S 4}$ \\
\hline Figure S4 & ${ }^{1} \mathrm{H}$ NMR of compound $\mathbf{3 b}$ recorded in $\mathrm{CDCl}_{3}$ & S5 \\
\hline Figure S5 & HRMS spectrum of compound $3 \mathbf{b}$ & S6 \\
\hline Figure S6 & ${ }^{13} \mathrm{C}$ NMR of compound $\mathbf{3 b}$ recorded in $\mathrm{CDCl}_{3}$ & S7 \\
\hline Figure S7 & ${ }^{1} \mathrm{H}$ NMR of compound $\mathbf{3 c}$ recorded in DMSO- $\mathrm{d}_{6}$ & $\mathbf{S 8}$ \\
\hline Figure S8 & HRMS spectrum of compound $\mathbf{3 c}$ & S9 \\
\hline Figure S9 & ${ }^{13} \mathrm{C}$ NMR of compound $\mathbf{3 c}$ recorded in DMSO- $\mathrm{d}_{6}$ & S10 \\
\hline Figure S10 & ${ }^{1} \mathrm{H}$ NMR of compound 4 recorded in $\mathrm{CDCl}_{3}$ & S11 \\
\hline Figure S11 & HRMS spectrum of compound 4 & S12 \\
\hline Figure S12 & ${ }^{13} \mathrm{C}$ NMRof compound 4 recorded in $\mathrm{CDCl}_{3}$ & S13 \\
\hline Figure S13 & ${ }^{1} \mathrm{HNMR}$ of compound $\mathbf{5}$ and $\mathbf{5}^{\prime}$ recorded in $\mathrm{CDCl}_{3}$ & S14 \\
\hline Figure S14 & HRMS spectrum of compound $\mathbf{5}$ and $\mathbf{5}^{\prime}$ & S15 \\
\hline Figure S15 & ${ }^{13} \mathrm{C}$ NMR of compound $\mathbf{5}$ and $\mathbf{5}^{\prime}$ recorded in $\mathrm{CDCl}_{3}$ & S16 \\
\hline Figure S16 & ${ }^{1} \mathrm{H}-1 \mathrm{H}$ NOESY spectrum of compound $\mathbf{5}^{+\mathbf{5}^{\prime}}$ recorded in $\mathrm{CDCl}_{3}$ & S17 \\
\hline Figure S17 & Cyclic voltamogram of compound $\mathbf{5}+\mathbf{5}^{\prime}$ & S18 \\
\hline Figure S18 & ${ }^{1} \mathrm{HNMR}$ of compound 6 recorded in $\mathrm{CDCl}_{3}$ & S19 \\
\hline Figure S19 & HRMS spectrum of compound $\mathbf{6}$ & $\mathbf{S 2 0}$ \\
\hline Figure S20 & ${ }^{13} \mathrm{C}$ NMR of compound $\mathbf{6}$ recorded in $\mathrm{CDCl}_{3}$ & S21 \\
\hline Figure S21 & $\begin{array}{l}\text { 1D and 2D NMR characterization of compound } 6 \text { recorded in } \\
\mathrm{CDCl}_{3}\end{array}$ & S22 \\
\hline Figure S22 & ${ }^{1} \mathrm{HNMR}$ of compound 7 recorded in $\mathrm{CDCl}_{3}$ & S23 \\
\hline Figure S23 & HRMS spectrum of compound 7 & $\mathbf{S 2 4}$ \\
\hline Figure S24 & ${ }^{13} \mathrm{C}$ NMR of compound 7 recorded in $\mathrm{CDCl}_{3}$ & $\mathbf{S 2 5}$ \\
\hline Table S1 & $\begin{array}{l}\text { Table of free thermal energy in normal and inverted } \\
\text { conformation of } \mathbf{2 a} \text { and } \mathbf{4 - 7}\end{array}$ & S26 \\
\hline $\begin{array}{l}\text { Figure S25- } \\
\text { Figure S33 }\end{array}$ & $\begin{array}{l}\text { Cartesian coordinates of the S0 optimized structures of the } \\
\text { compounds } \mathbf{2 a} \text { and } \mathbf{4 - 7} \text { in their both the conformation (normal } \\
\text { and inverted) }\end{array}$ & S27 \\
\hline
\end{tabular}



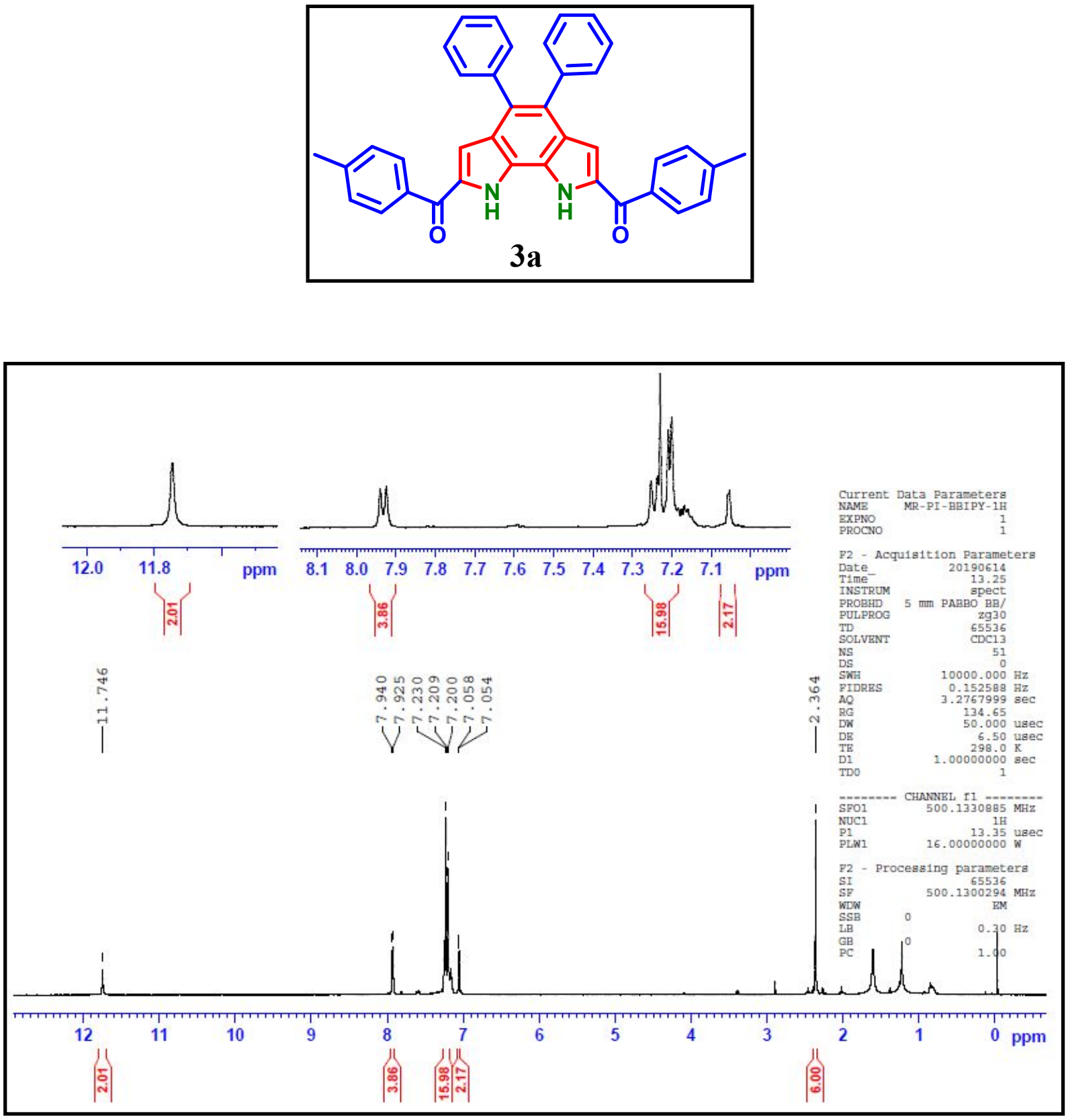

Figure S1. ${ }^{1} \mathrm{H}$ NMR of compound 3 a recorded in $\mathrm{CDCl}_{3}$ at $25^{\circ} \mathrm{C} .(500 \mathrm{MHz})$ 


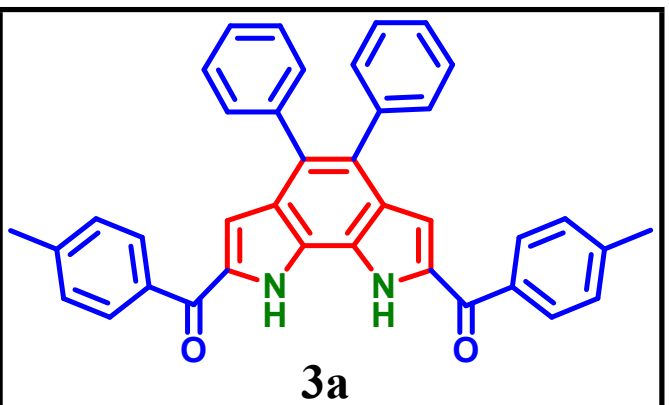

Chemical Formula: $\mathrm{C}_{38} \mathrm{H}_{28} \mathrm{~N}_{2} \mathrm{O}_{2}$ Exact Mass: 544.2151

Observed Mass $=545.2227[\mathrm{M}+\mathrm{H}]^{+}$

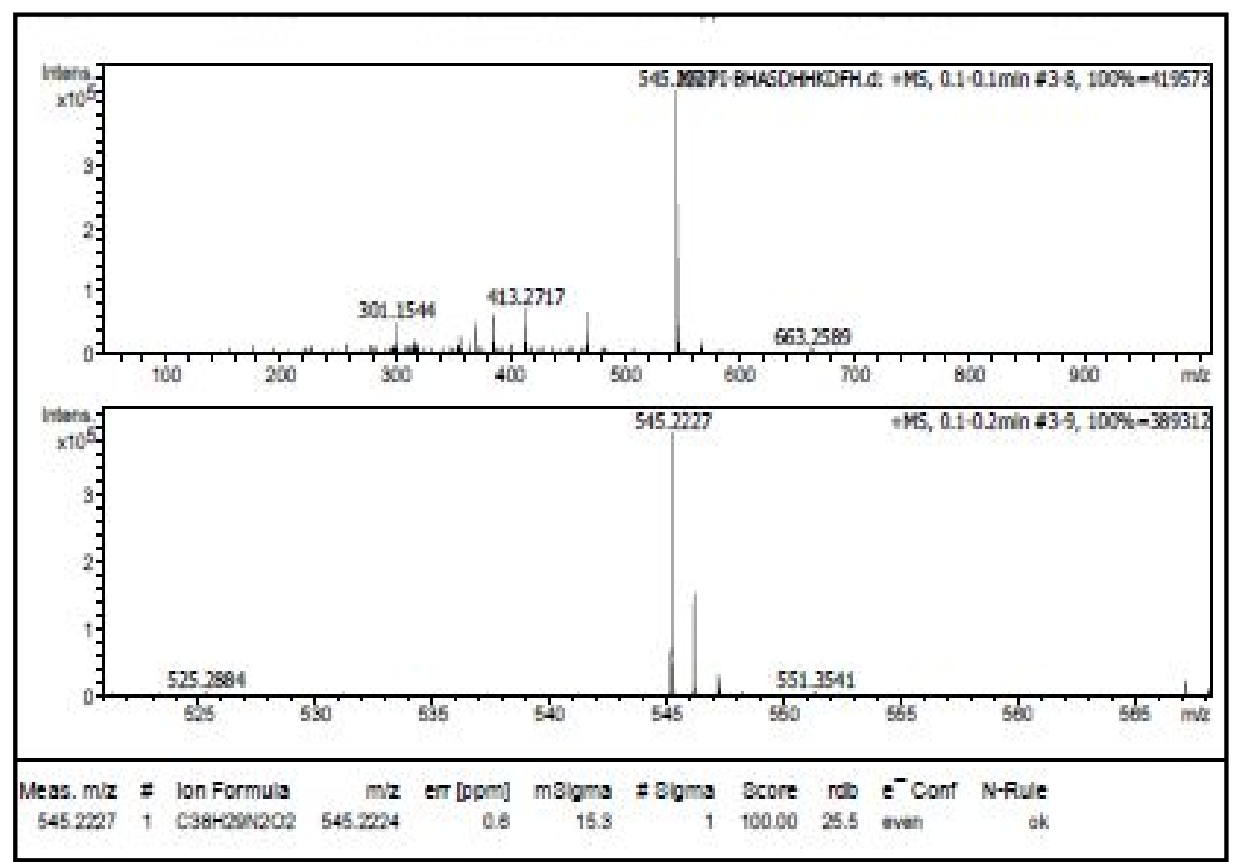

Figure S2. HRMS spectrum of compound 3a. 

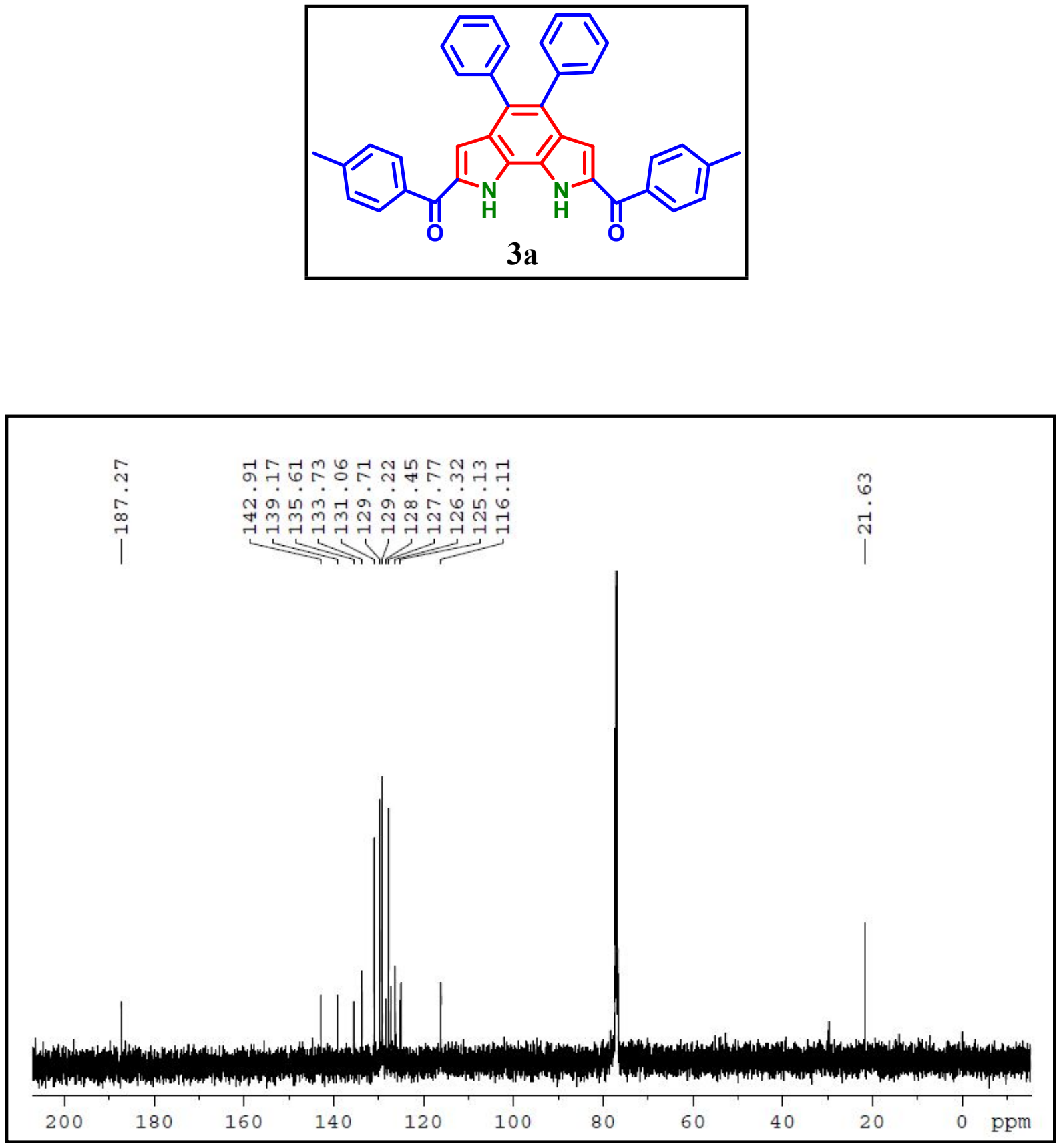

Figure S3. ${ }^{13} \mathrm{C}\{1 \mathrm{H}\}$ NMR of compound 3a recorded in $\mathrm{CDCl}_{3}$ at $25{ }^{\circ} \mathrm{C}(125 \mathrm{MHz})$. 

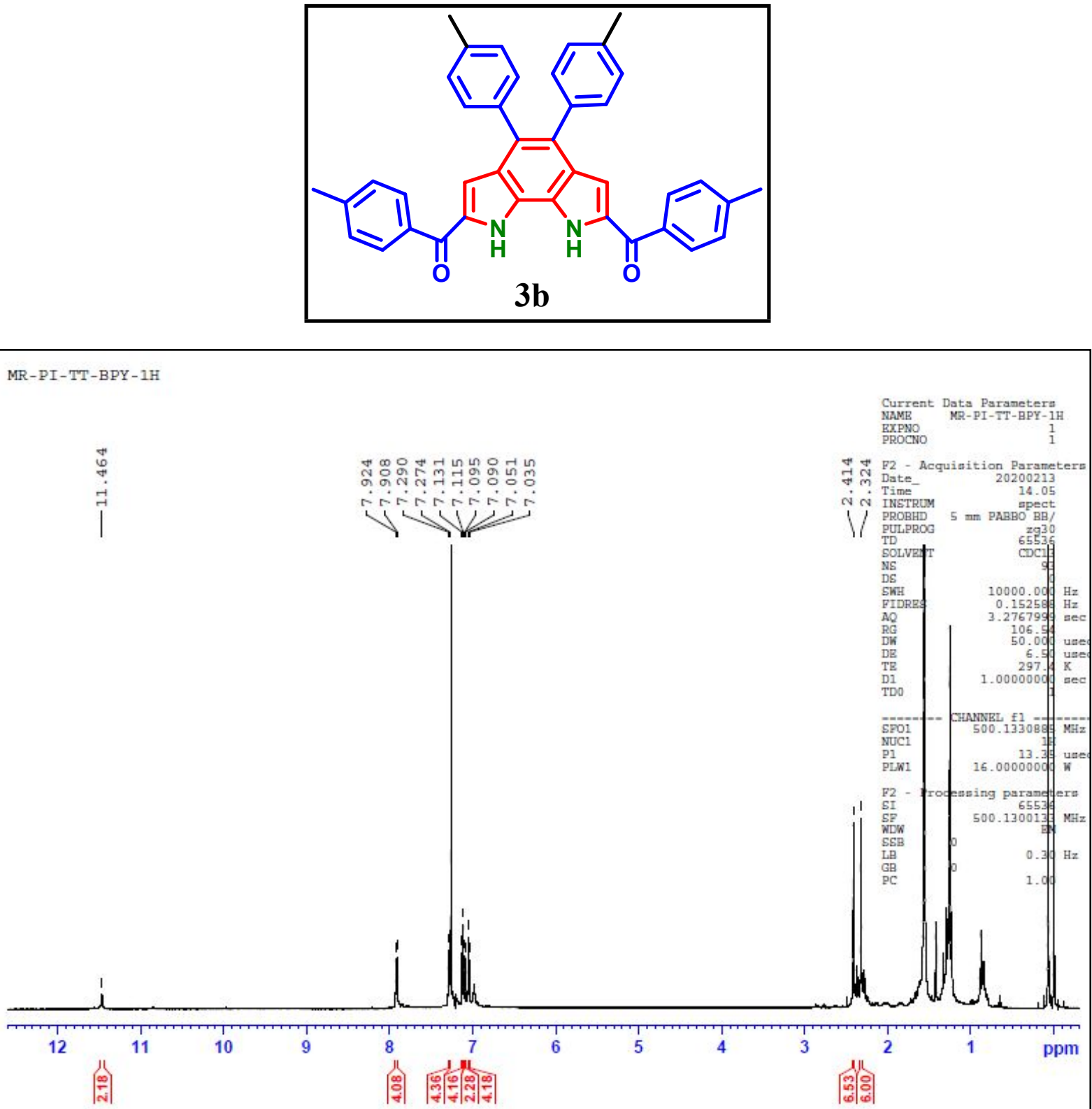

Figure S4. ${ }^{1} \mathrm{H}$ NMR of compound $\mathbf{3 b}$ recorded in $\mathrm{CDCl}_{3}$ at $25{ }^{\circ} \mathrm{C} .(500 \mathrm{MHz})$. 


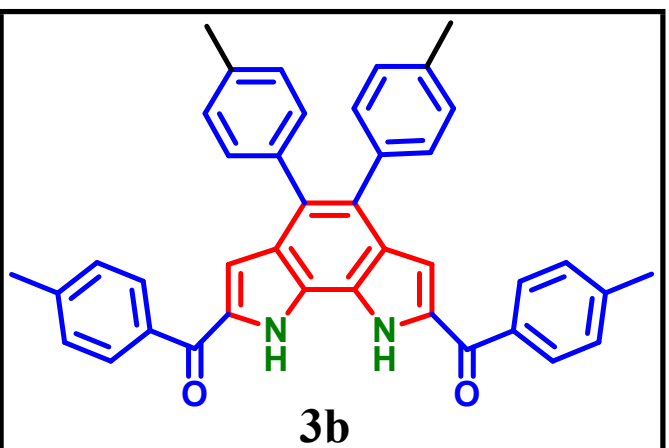

Chemical Formula: $\mathrm{C}_{40} \mathrm{H}_{32} \mathrm{~N}_{2} \mathrm{O}_{2}$ Exact Mass: 572.2464

\section{Observed Mass $=573.2543[\mathrm{M}+\mathrm{H}]^{+}$}

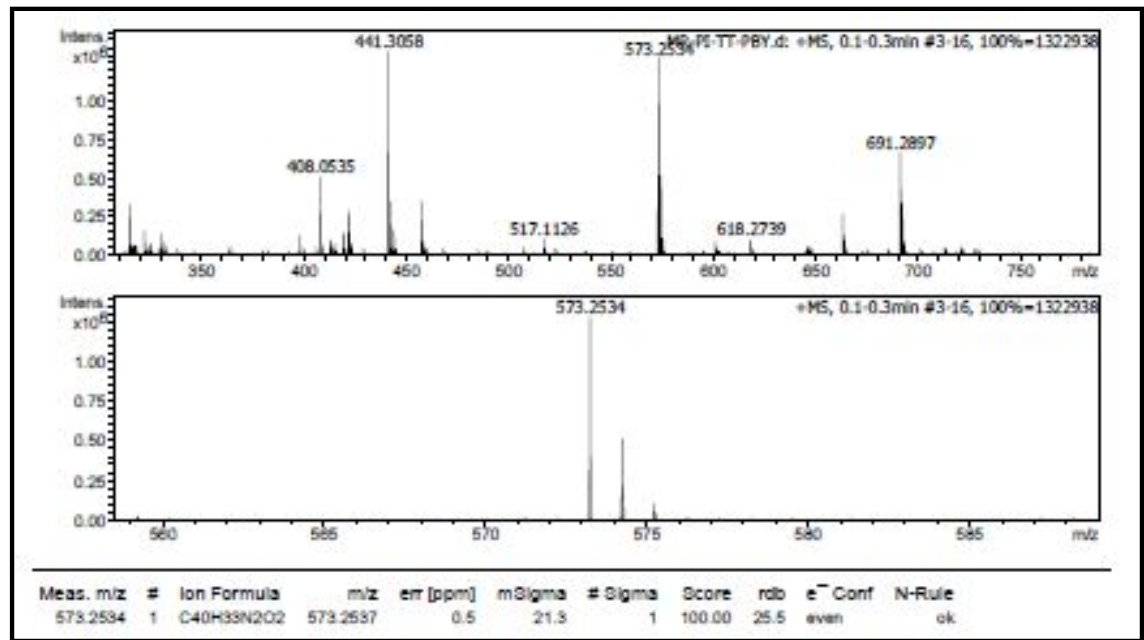

Figure S5. HRMS spectrum of compound $\mathbf{3 b}$. 

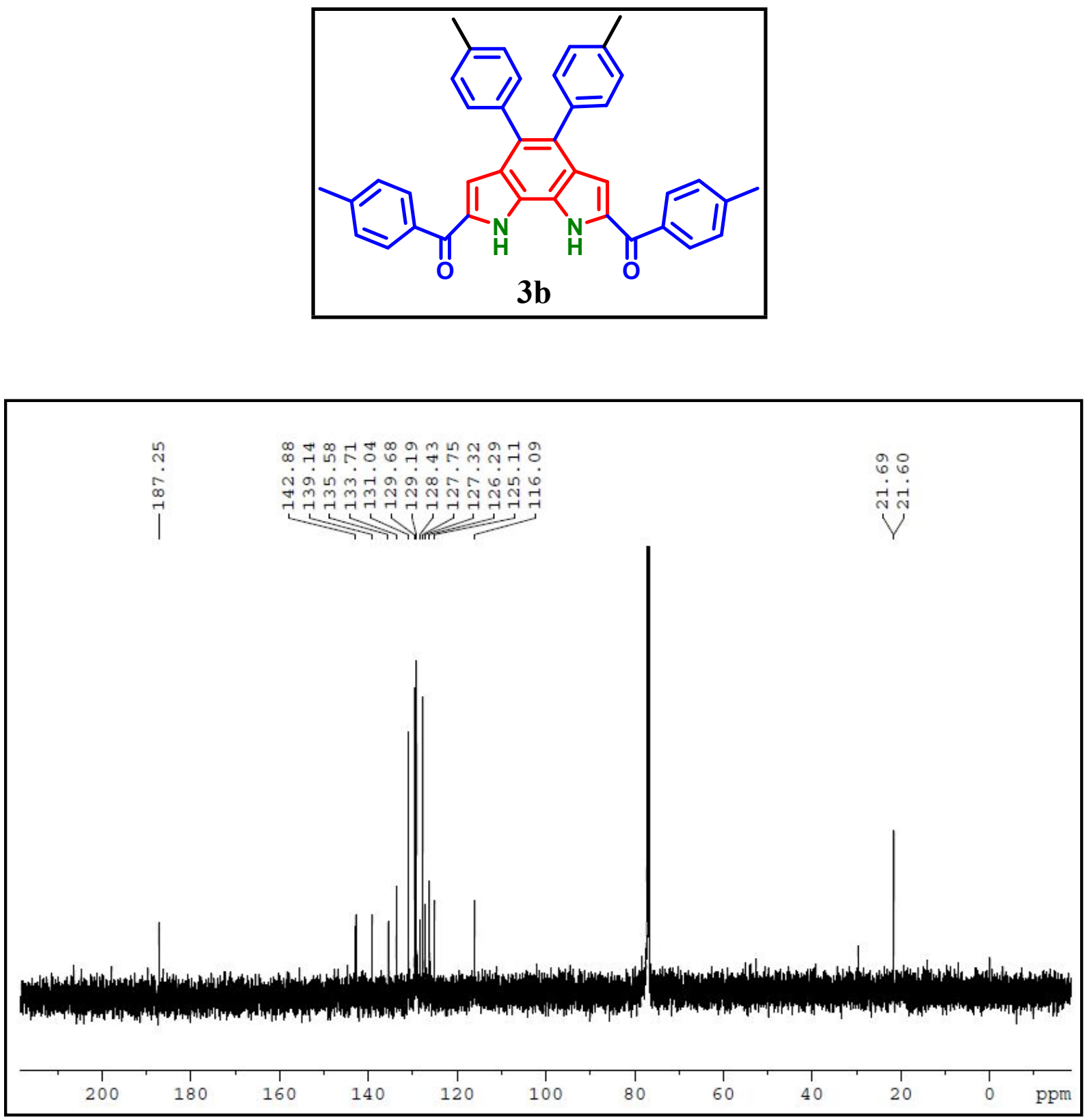

Figure S6. ${ }^{13} \mathrm{C}\left\{{ }^{1} \mathrm{H}\right\} \mathrm{NMR}$ of compound $\mathbf{3 b}$ recorded in $\mathrm{CDCl}_{3}$ at $25{ }^{\circ} \mathrm{C}(125 \mathrm{MHz})$. 

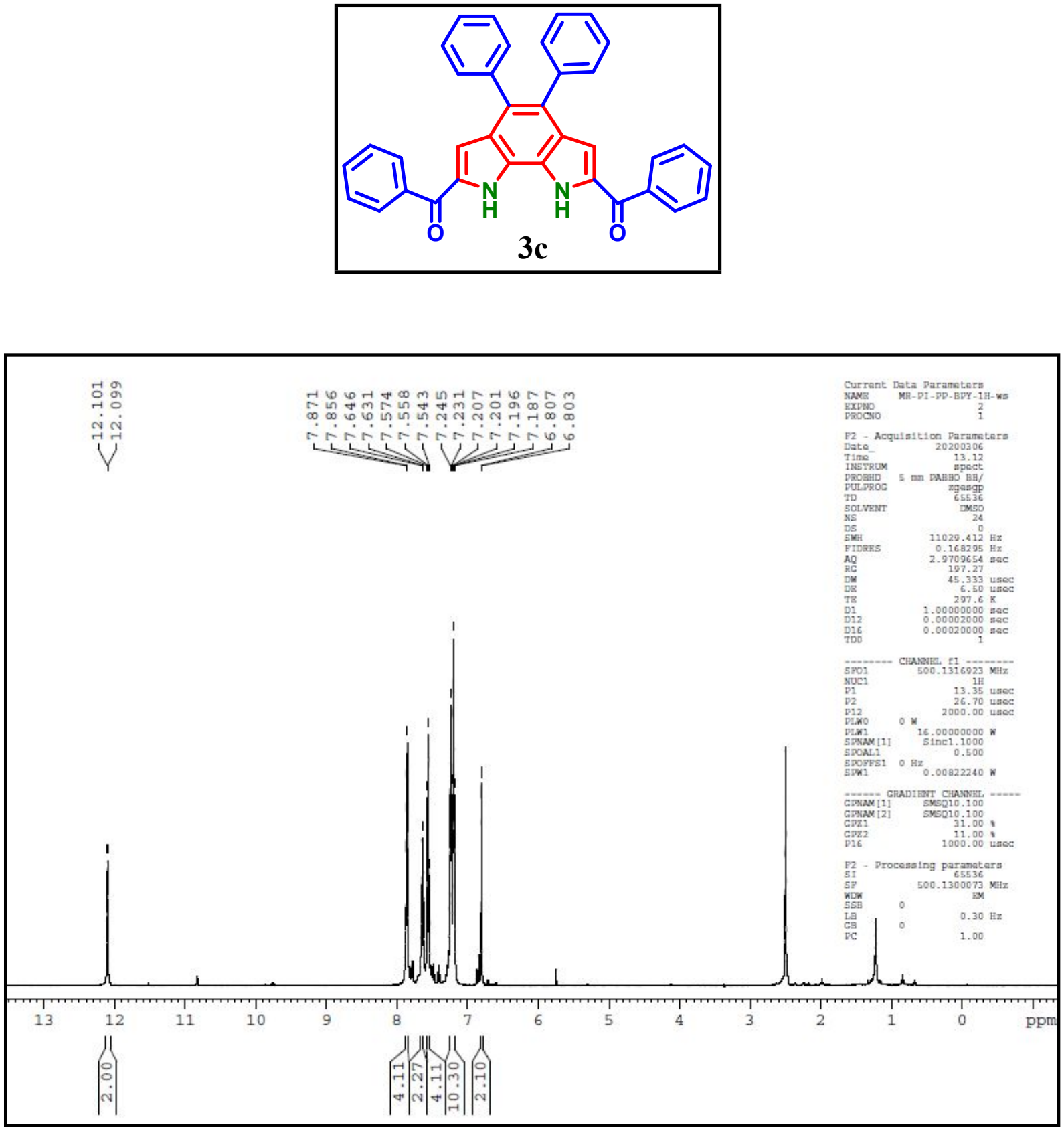

Figure S7. ${ }^{1} \mathrm{H}$ NMR of compound 3c recorded in DMSO-d ${ }_{6}$ at $25{ }^{\circ} \mathrm{C}(500 \mathrm{MHz})$. 


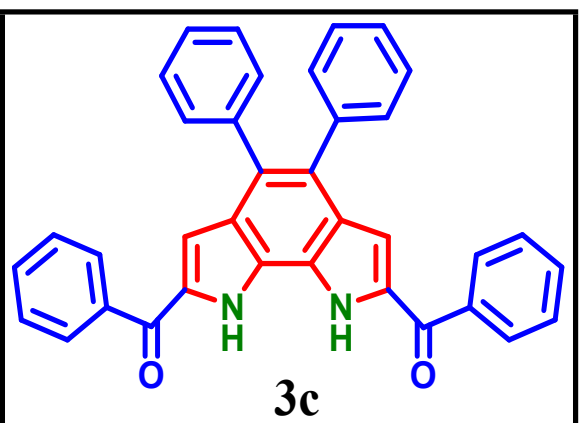

Chemical Formula: $\mathrm{C}_{36} \mathrm{H}_{24} \mathrm{~N}_{2} \mathrm{O}_{2}$ Exact Mass: 516.1838

Observed Mass= $517.1918[\mathrm{M}+\mathrm{H}]^{+}$

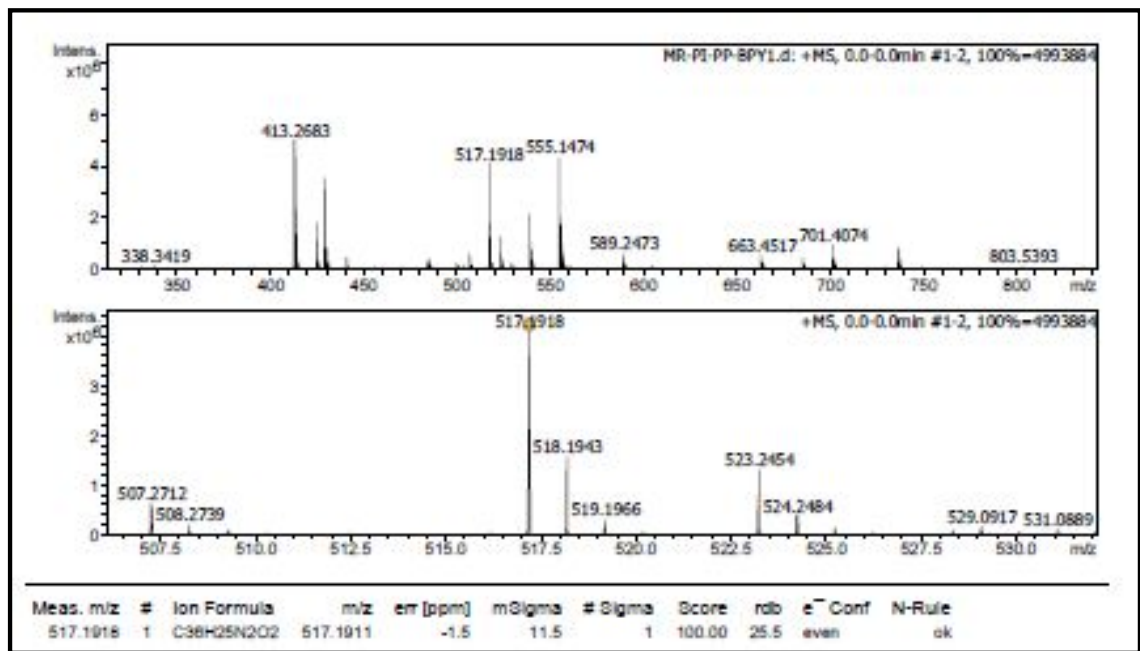

Figure S8. HRMS spectrum of compound 3c. 

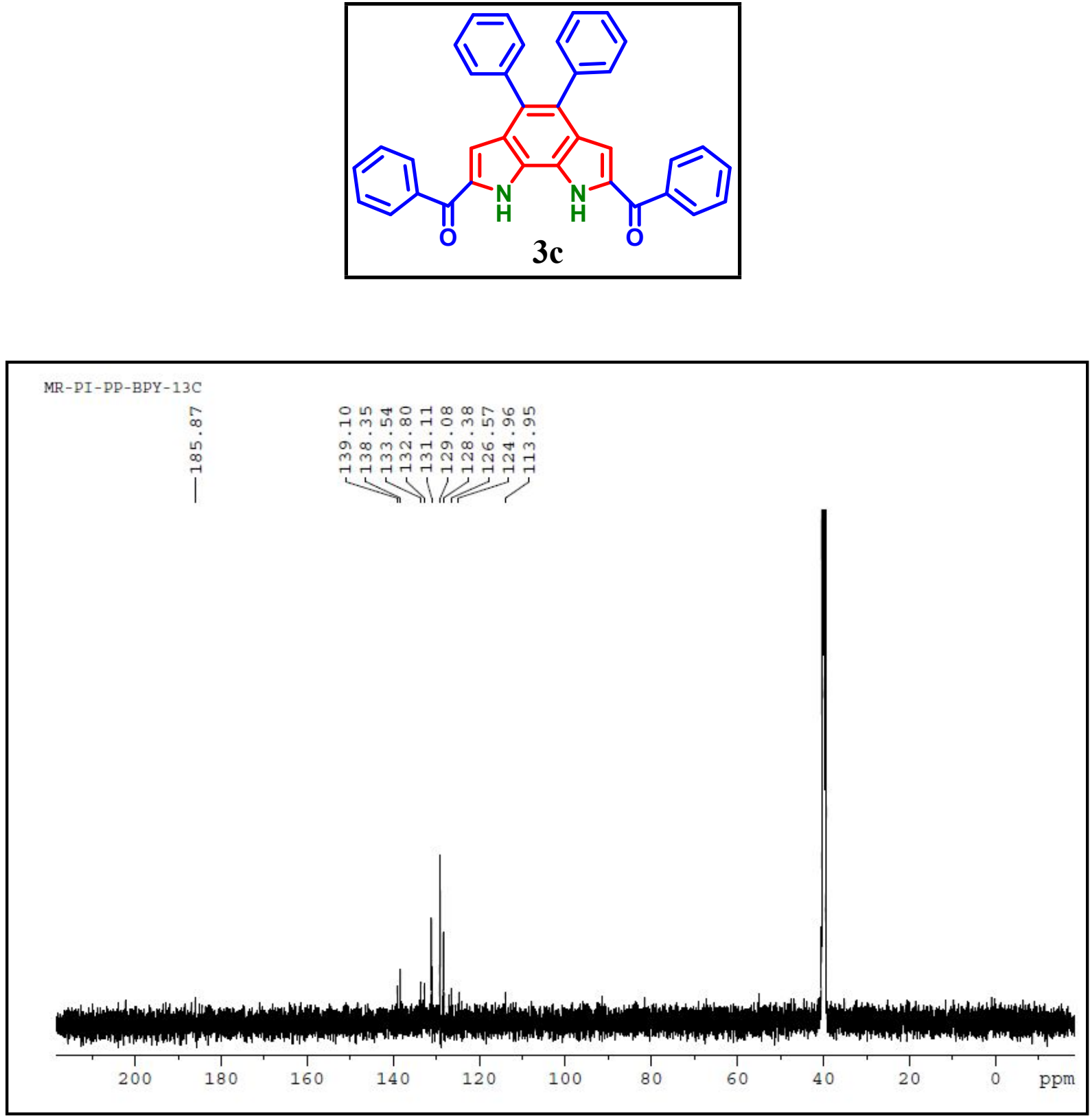

Figure S9. ${ }^{13} \mathrm{C}\left\{{ }^{1} \mathrm{H}\right\}$ NMR of compound 3c recorded in DMSO- $\mathrm{d}_{6}$ at $25{ }^{\circ} \mathrm{C}(125 \mathrm{MHz})$. 

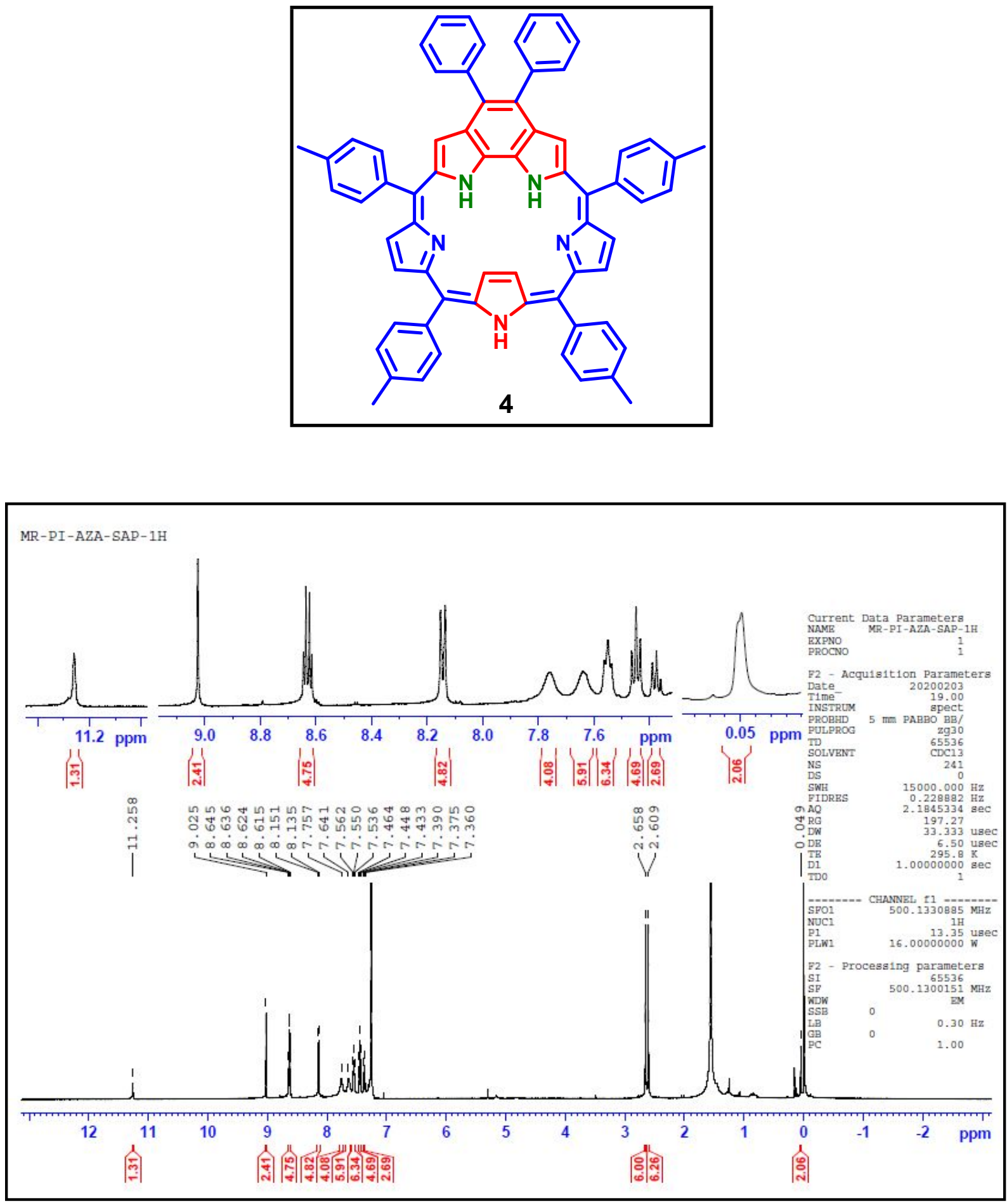

Figure S10. ${ }^{1} \mathrm{H}$ NMR of compound 4 recorded in $\mathrm{CDCl}_{3}$ at $25{ }^{\circ} \mathrm{C}(500 \mathrm{MHz})$. 


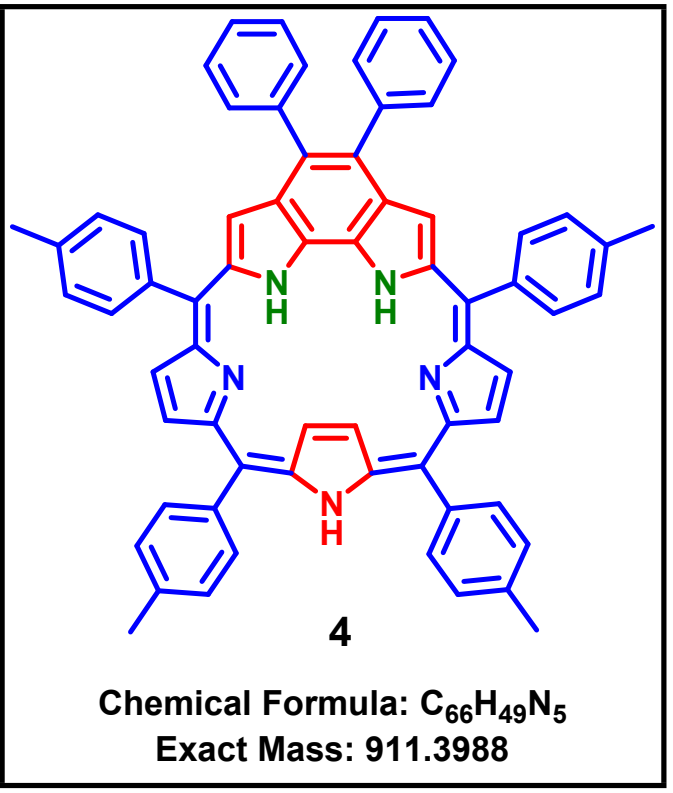

Observed Mass= $912.4061[\mathrm{M}+\mathrm{H}]^{+}$

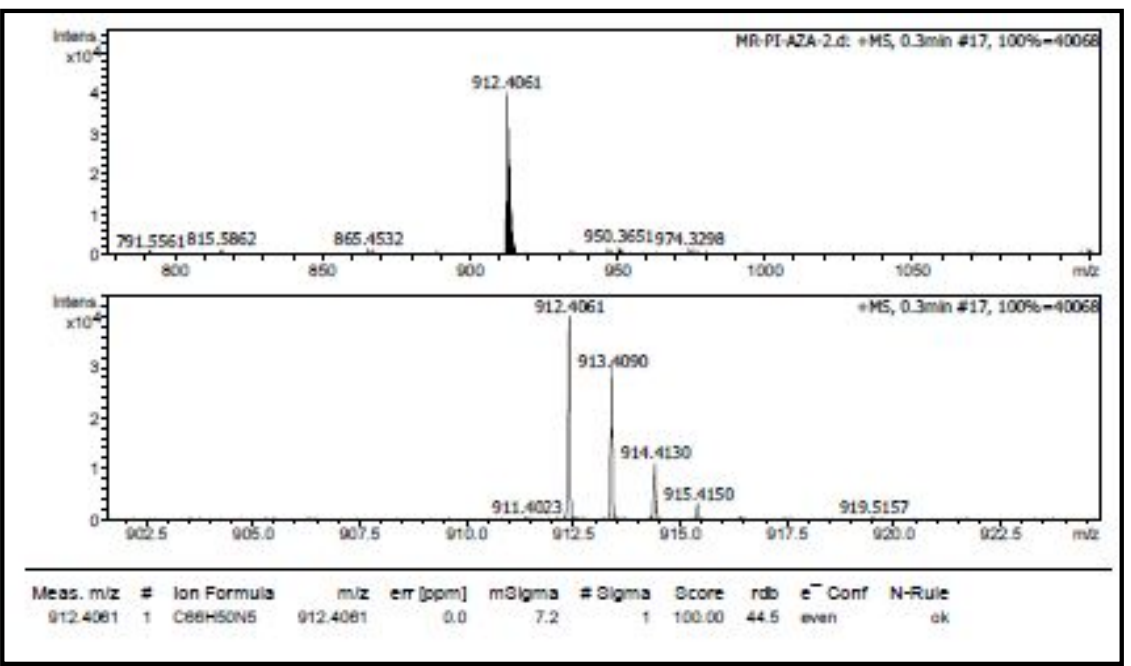

Figure S11. HRMS spectrum of compound 4. 

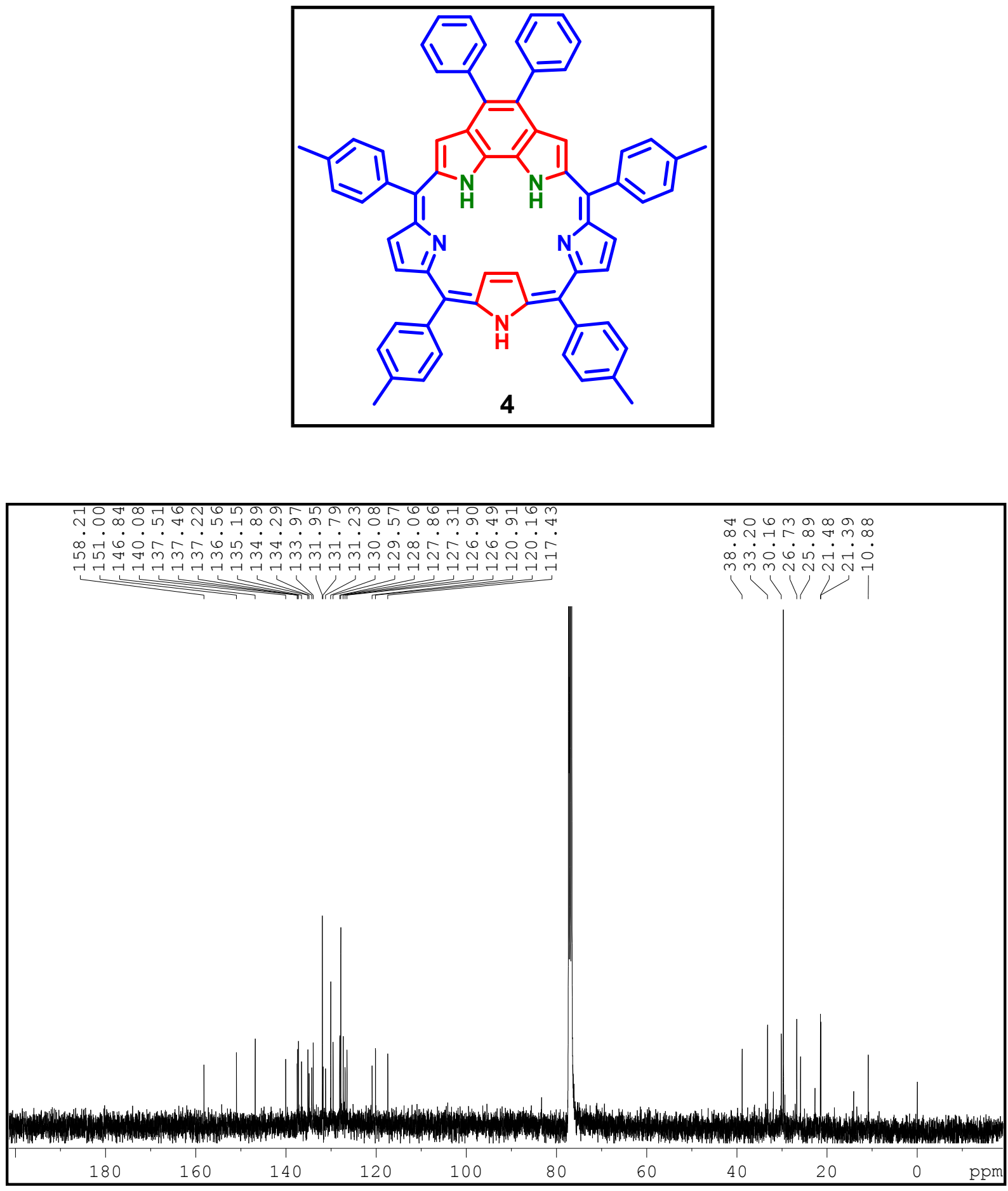

Figure S12. ${ }^{13} \mathrm{C}\left\{{ }^{1} \mathrm{H}\right\} \mathrm{NMR}$ of compound 4 recorded in $\mathrm{CDCl}_{3}$ at $25{ }^{\circ} \mathrm{C}(125 \mathrm{MHz})$.. 

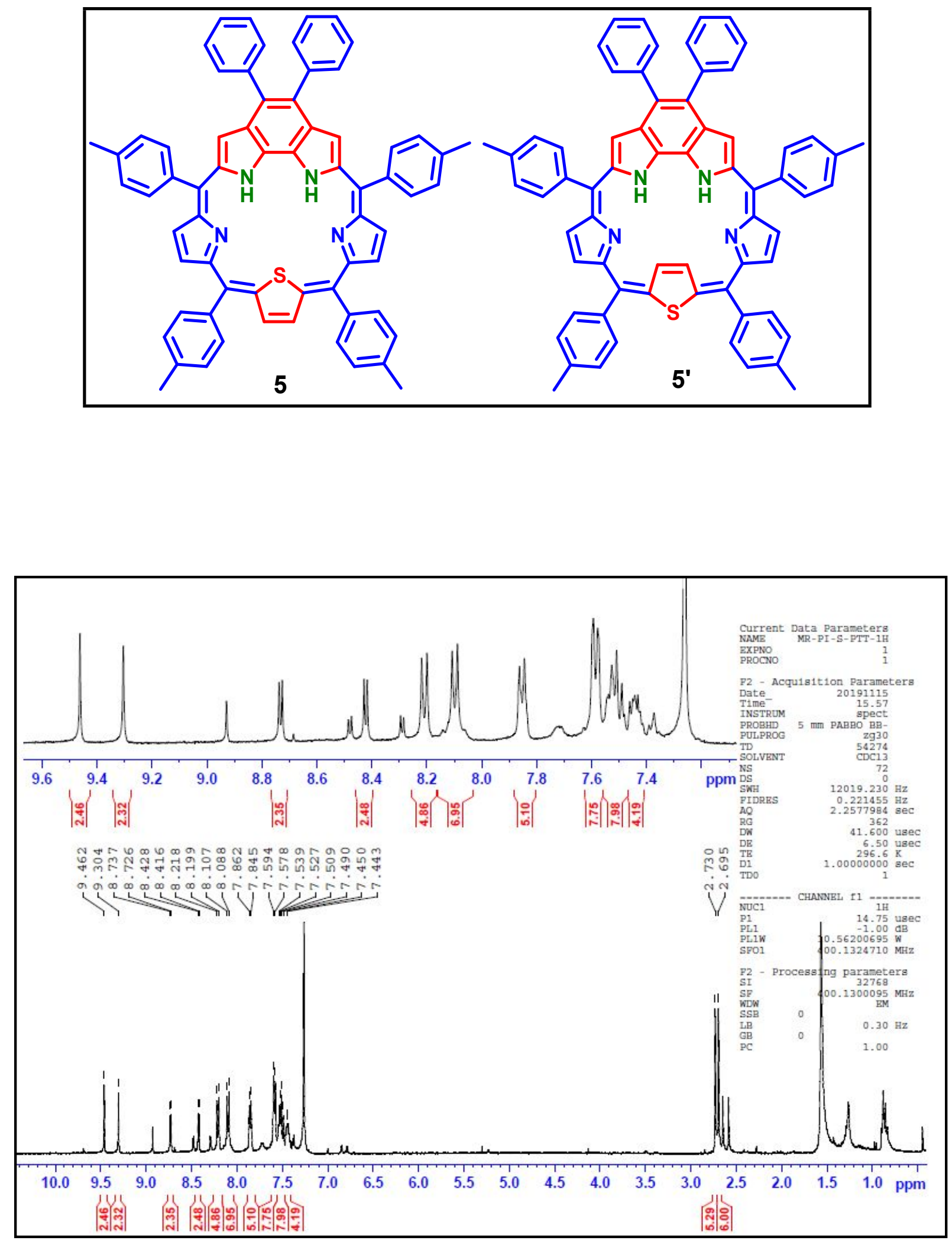

Figure S13. ${ }^{1} \mathrm{H}$ NMR of compound $\mathbf{5}^{+5^{\prime}}$ recorded in $\mathrm{CDCl}_{3}$ at $25^{\circ} \mathrm{C}(400 \mathrm{MHz})$. 


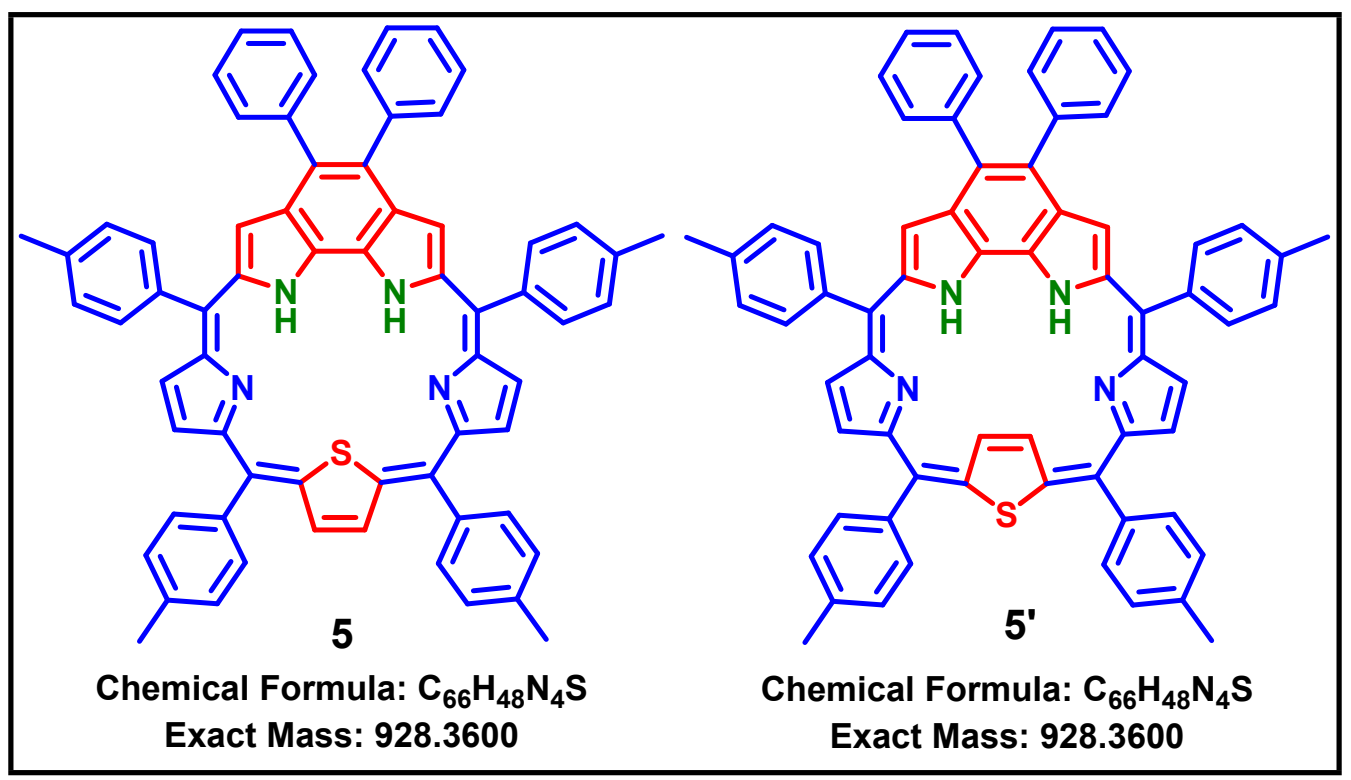

Observed Mass= $929.3679[\mathrm{M}+\mathrm{H}]^{+}$

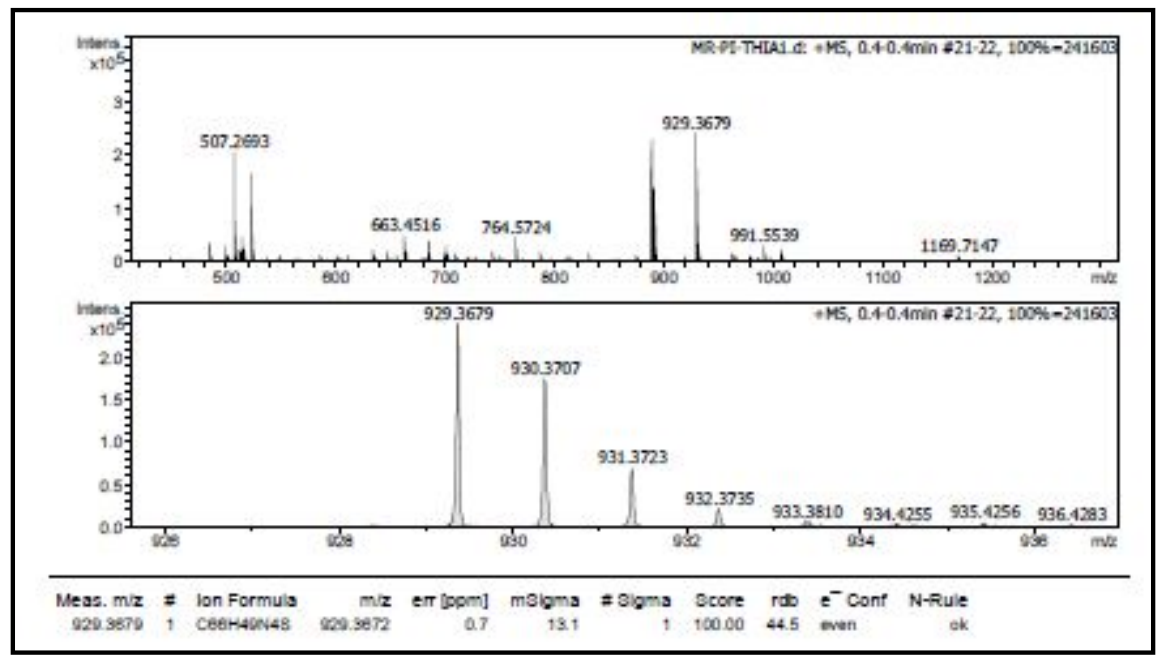

Figure S14. HRMS spectrum of compound $\mathbf{5}+\mathbf{5}^{\prime}$. 

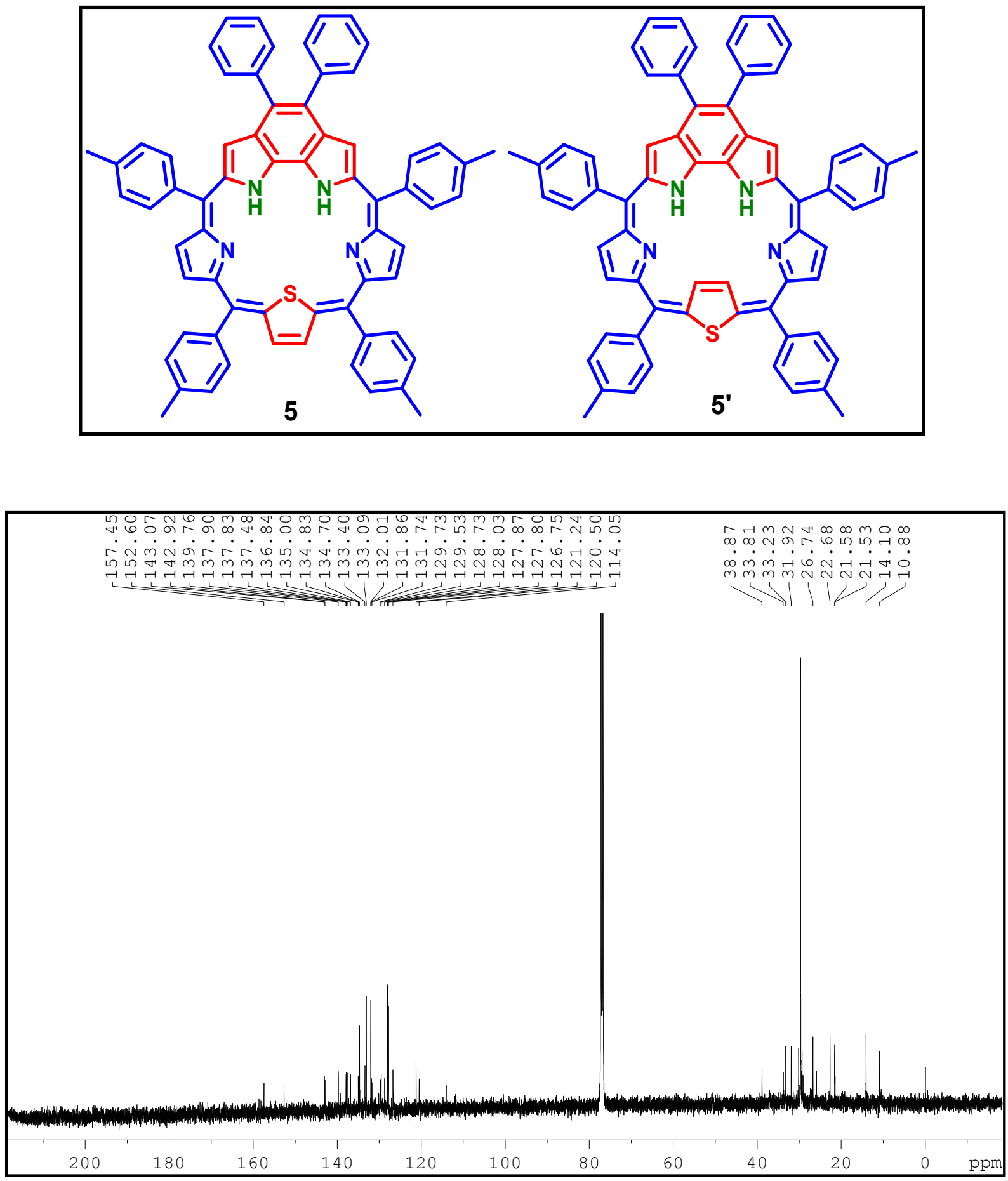

Figure S15. ${ }^{13} \mathrm{C}\left\{{ }^{1} \mathrm{H}\right\} \mathrm{NMR}$ of compound $\mathbf{5}^{+\mathbf{5}^{\prime}}$ recorded in $\mathrm{CDCl}_{3}$ at $25^{\circ} \mathrm{C}(100 \mathrm{MHz})$. 

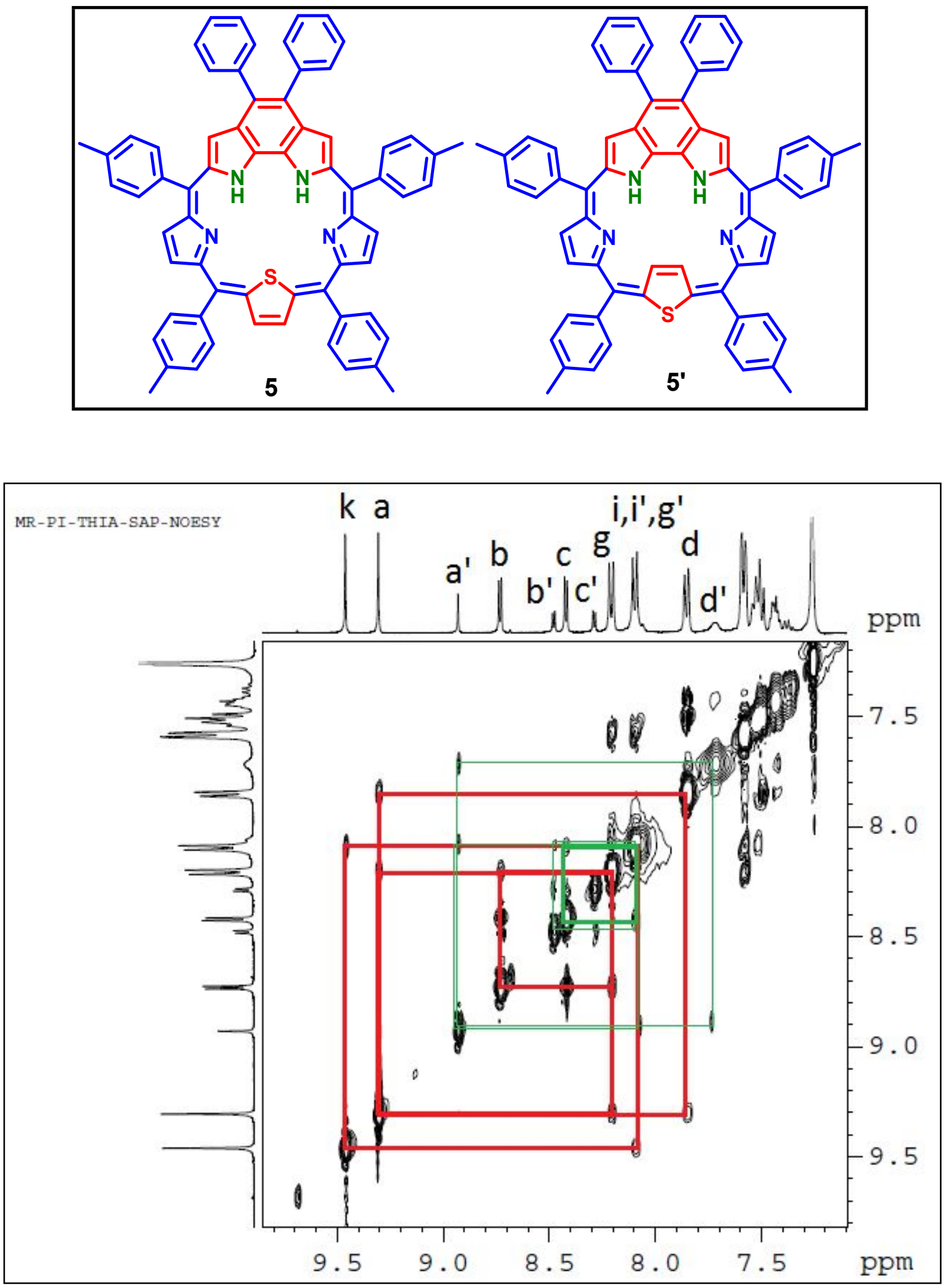

Figure S16. ${ }^{1} \mathrm{H}-1 \mathrm{H}$ NOESY spectrum of compound $\mathbf{5}^{+\mathbf{5}^{\prime}}$ recorded in $\mathrm{CDCl}_{3}$ at $25^{\circ} \mathrm{C}(400$ $\mathrm{MHz})$. 


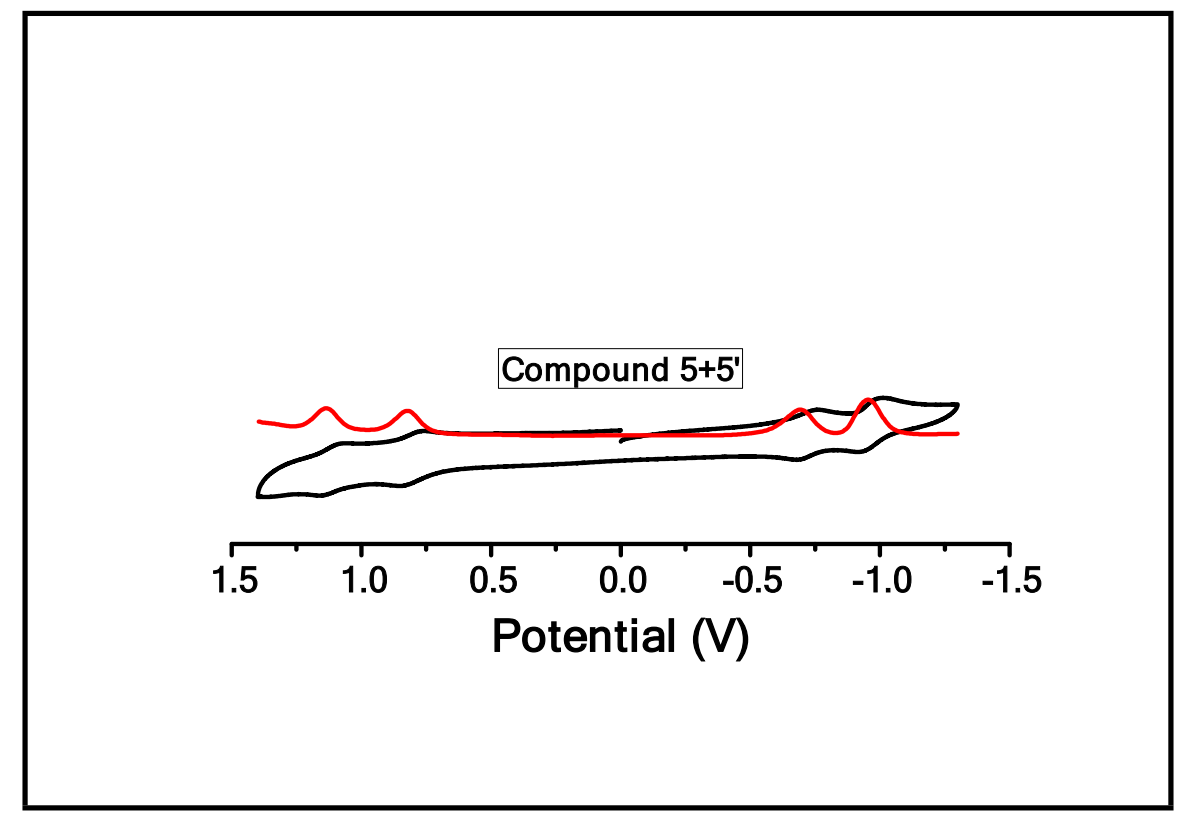

Figure S17: Cyclic voltammogram of compounds $\mathbf{5}^{\mathbf{+}} \mathbf{5}$ (black line) along with differential pulse voltammogram (red line), recorded in $\mathrm{CH}_{2} \mathrm{Cl}_{2}$ containing 0.1MTBAP as the supporting electrolyte and a saturated calomel electrode (SCE) as the reference electrode at scan rates of $50 \mathrm{mV} \mathrm{s}^{-1}$. 

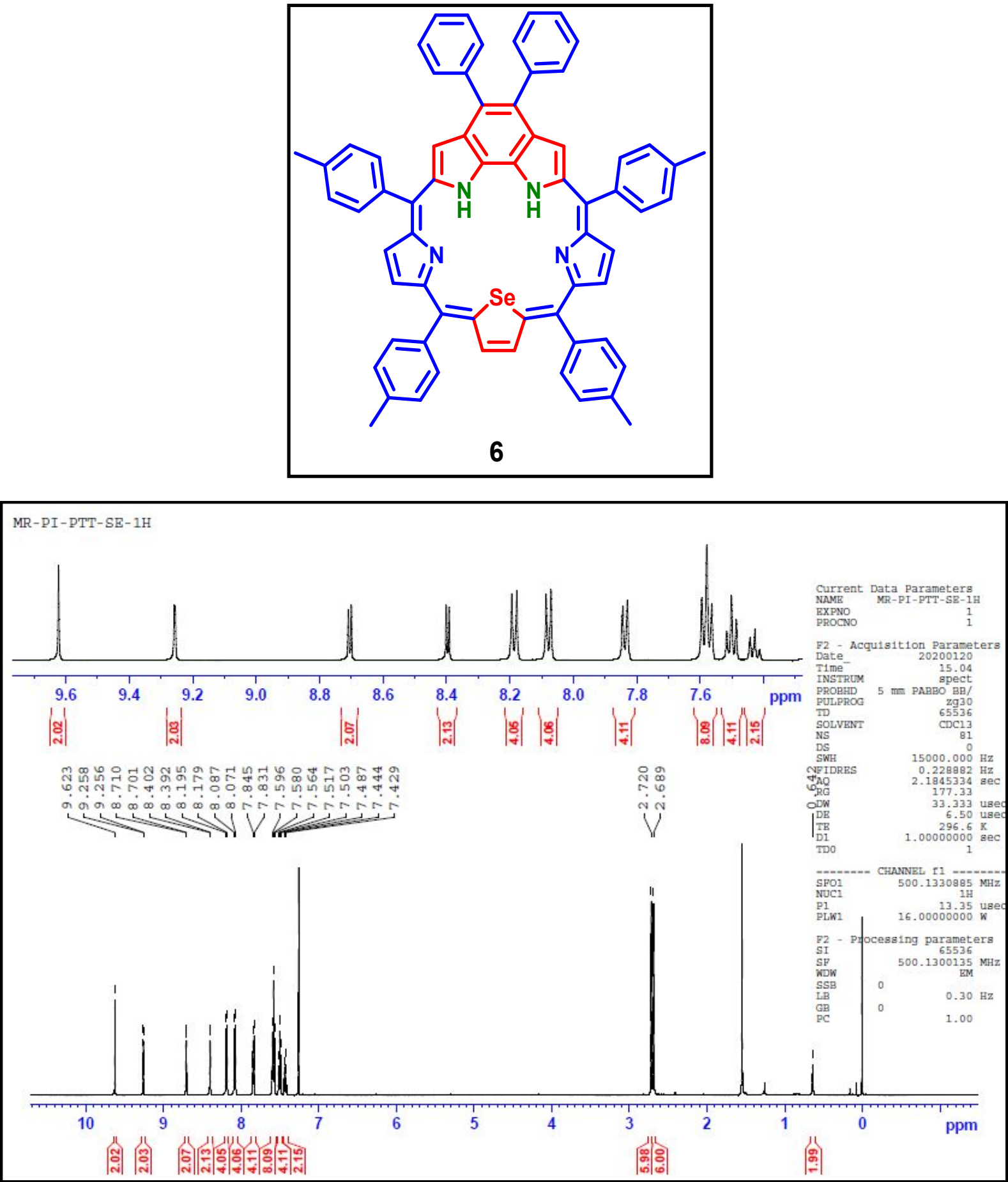

Figure S18. ${ }^{1} \mathrm{H}$ NMR of compound 6 recorded in $\mathrm{CDCl}_{3}$ at $25{ }^{\circ} \mathrm{C}(500 \mathrm{MHz})$. 


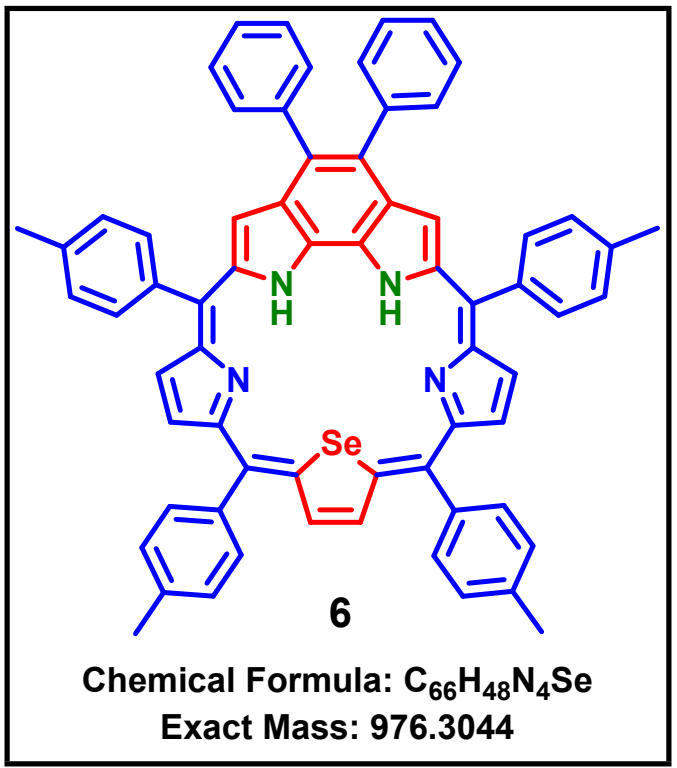

Observed Mass= $977.3126[\mathrm{M}+\mathrm{H}]^{+}$

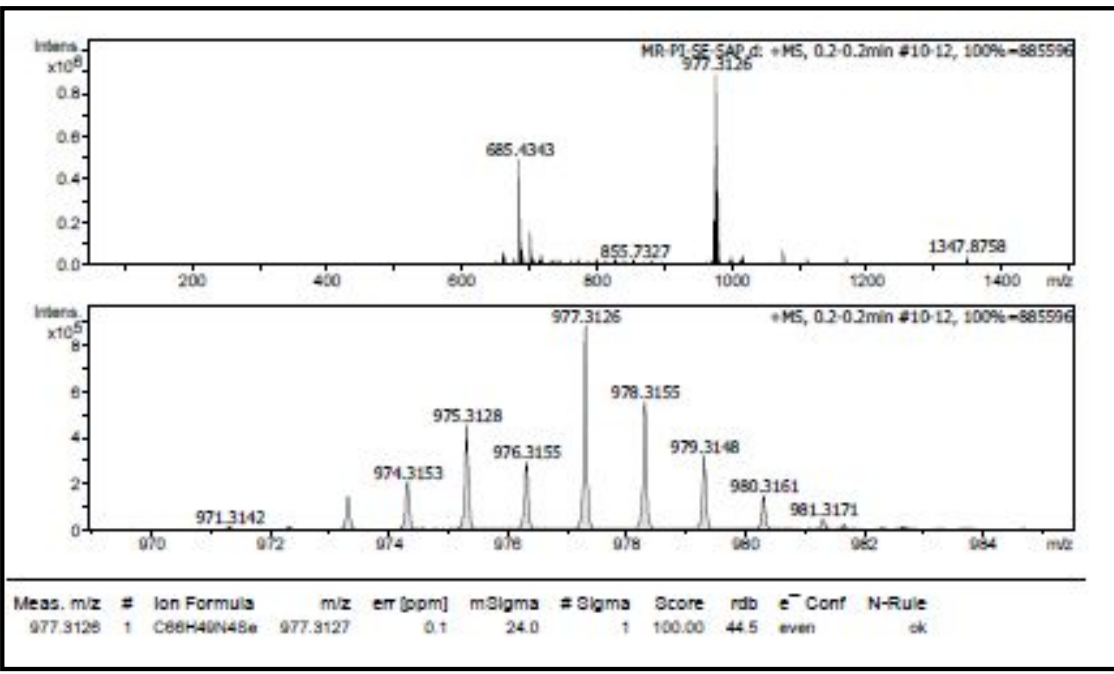

Figure S19. HRMS spectrum of compound 6. 

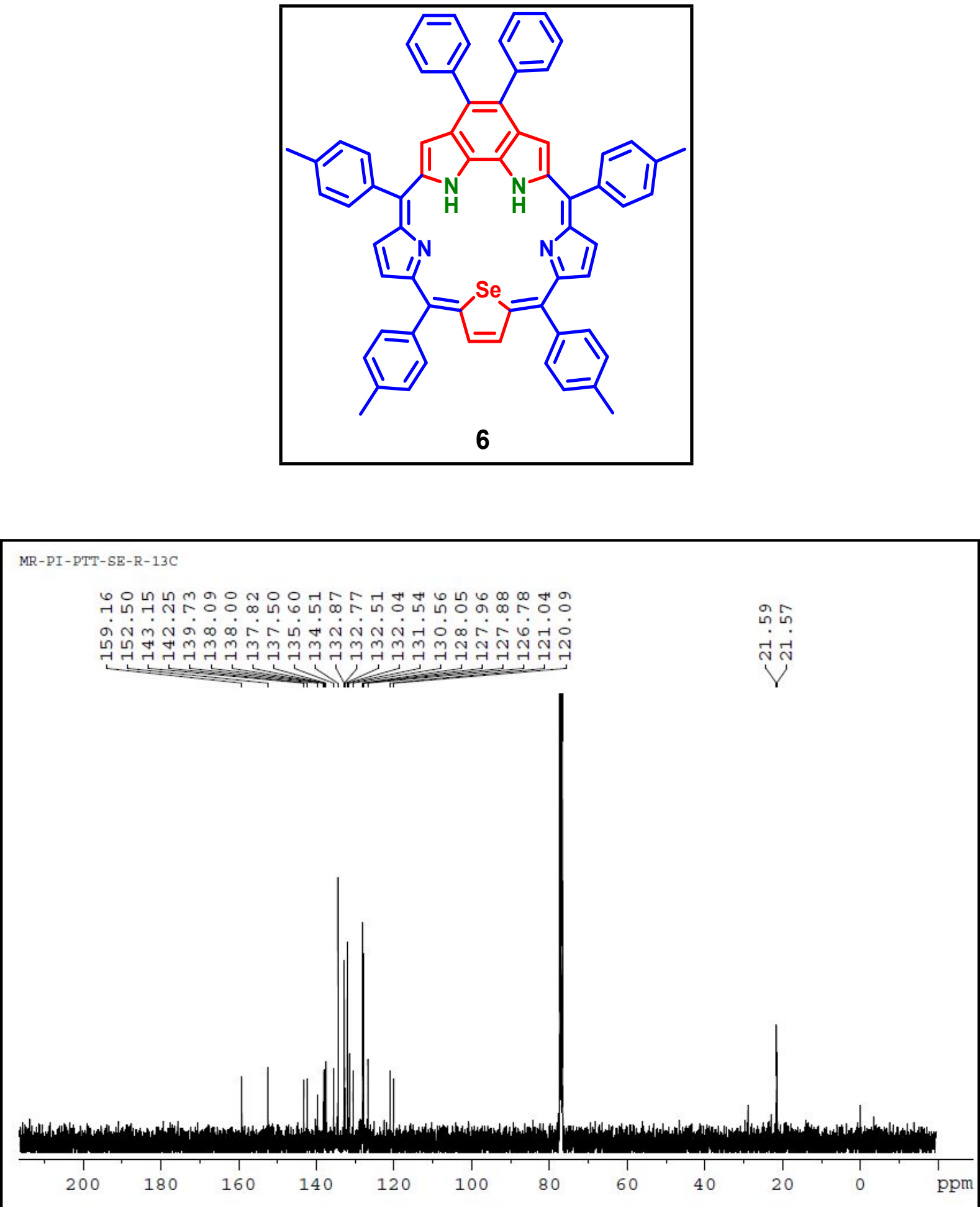

Figure S20. ${ }^{13} \mathrm{C}\left\{{ }^{1} \mathrm{H}\right\} \mathrm{NMR}$ of compound 6 recorded in $\mathrm{CDCl}_{3}$ at $25{ }^{\circ} \mathrm{C}(125 \mathrm{MHz})$.. 

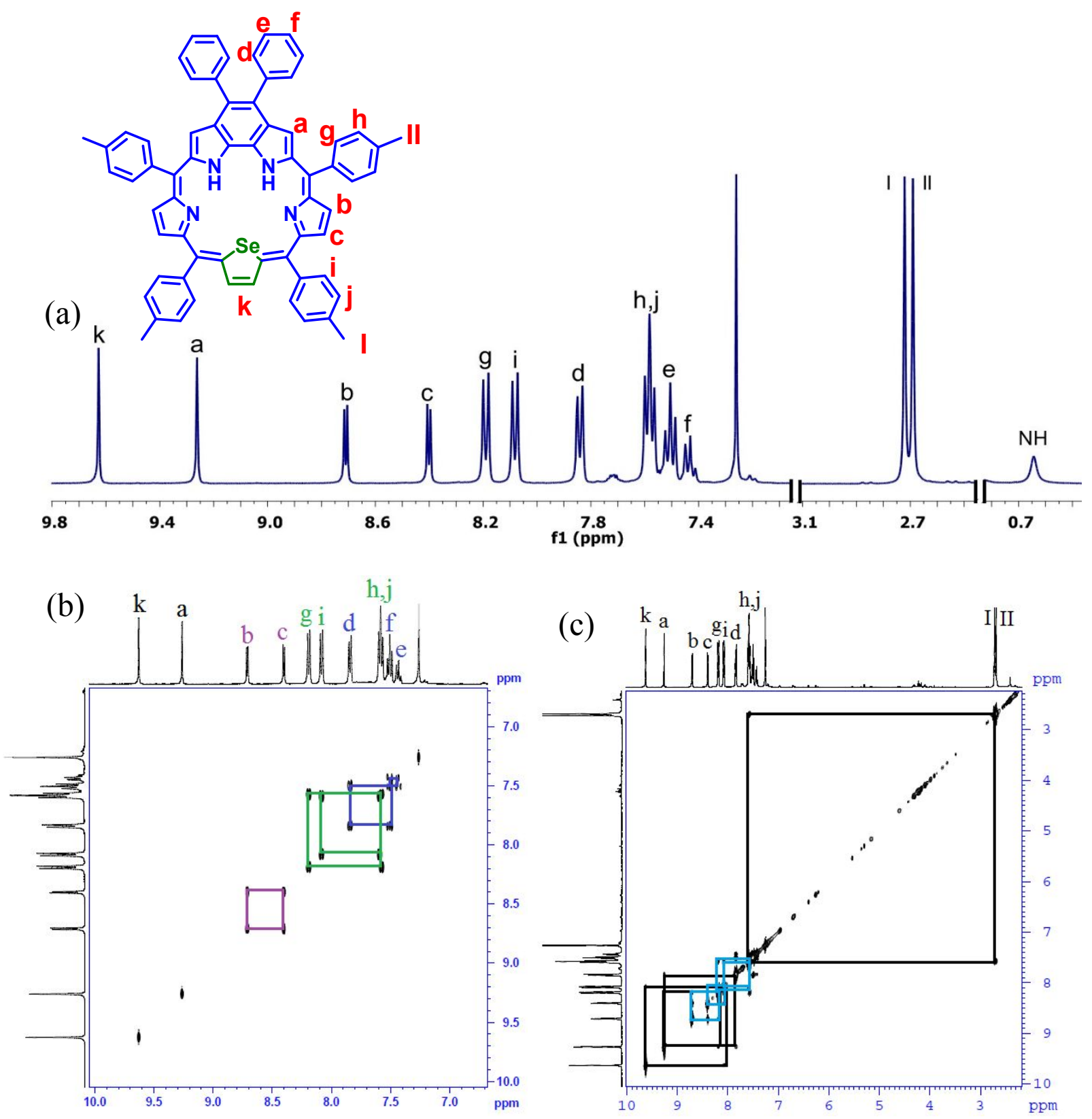

Figure S21:(a) Partial ${ }^{1} \mathrm{H}$ NMR, (b) ${ }^{1} \mathrm{H}-{ }^{1} \mathrm{H}$ COSY and (c) ${ }^{1} \mathrm{H}-{ }^{1} \mathrm{H}$ NOESY NMRspectraof macrocycle 6 recorded in $\mathrm{CDCl}_{3}$ at $25^{\circ} \mathrm{C}(500 \mathrm{MHz})$. 

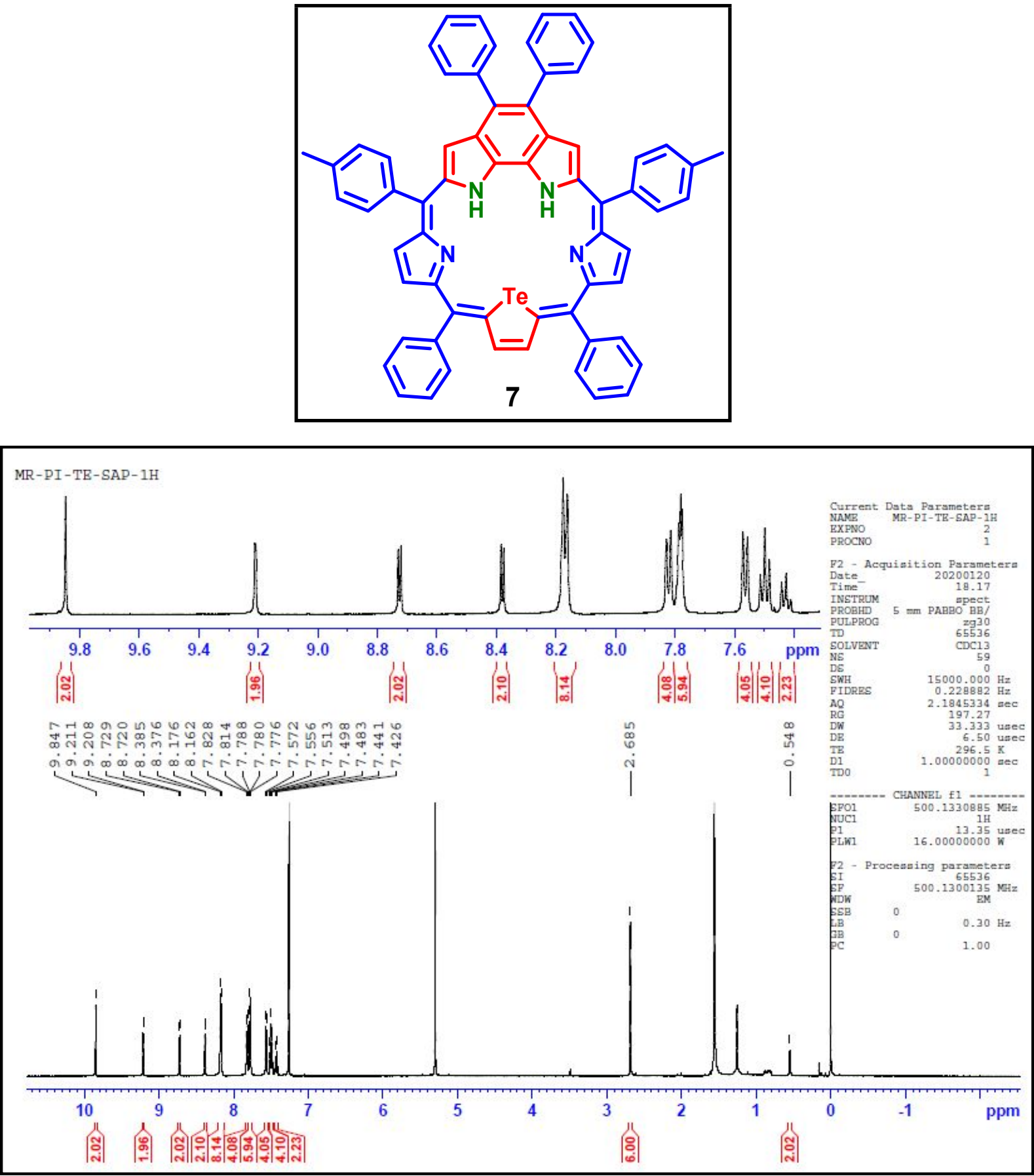

Figure S22. ${ }^{1} \mathrm{H}$ NMR of compound 7 recorded in $\mathrm{CDCl}_{3}$ at $25{ }^{\circ} \mathrm{C}(500 \mathrm{MHz})$. 


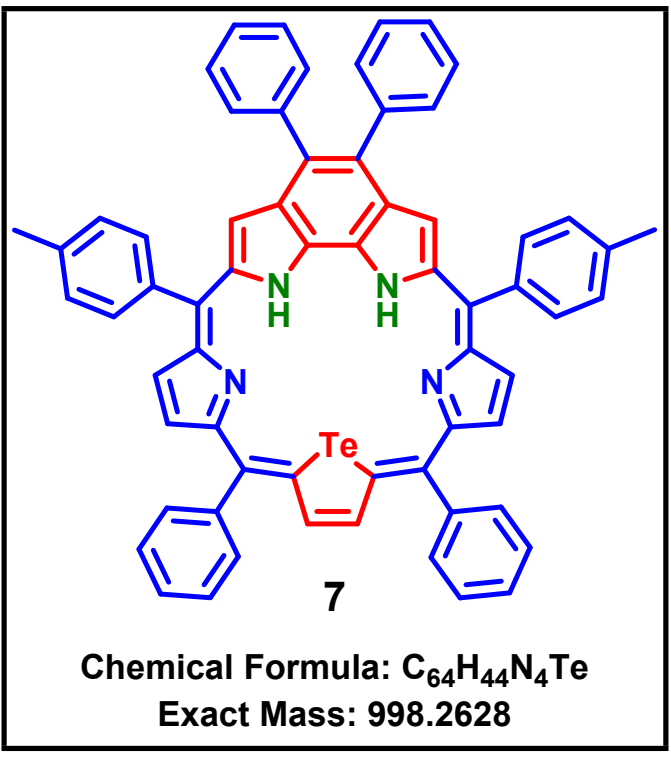

Observed Mass= $999.2708[\mathrm{M}+\mathrm{H}]^{+}$

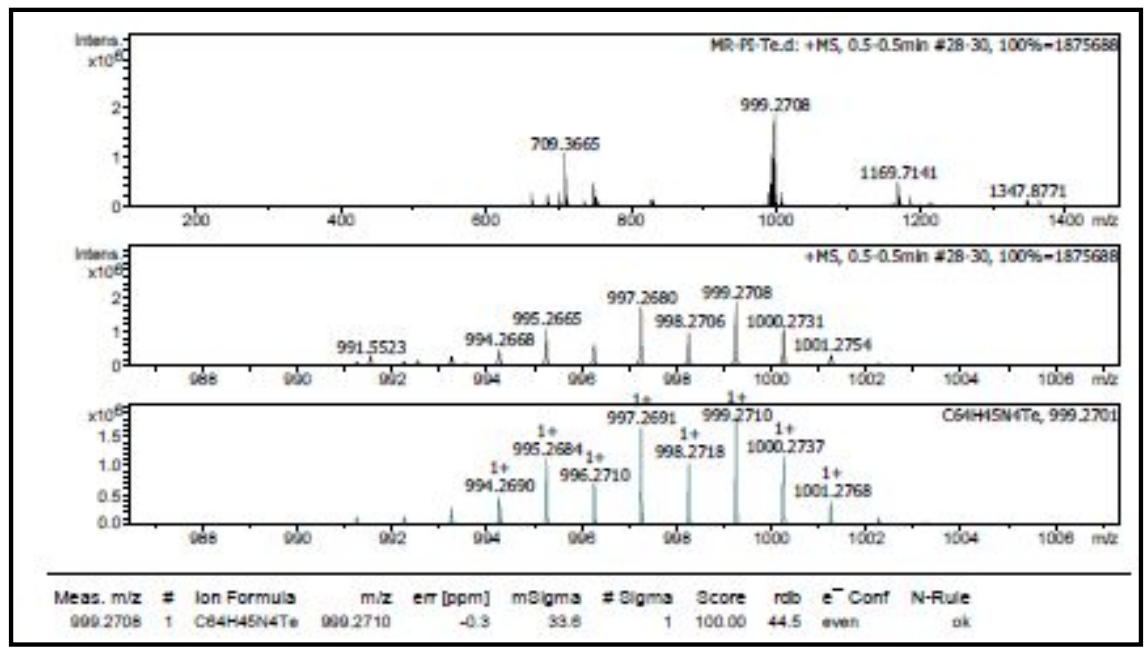

Figure S23. HRMS spectrum of compound 7. 

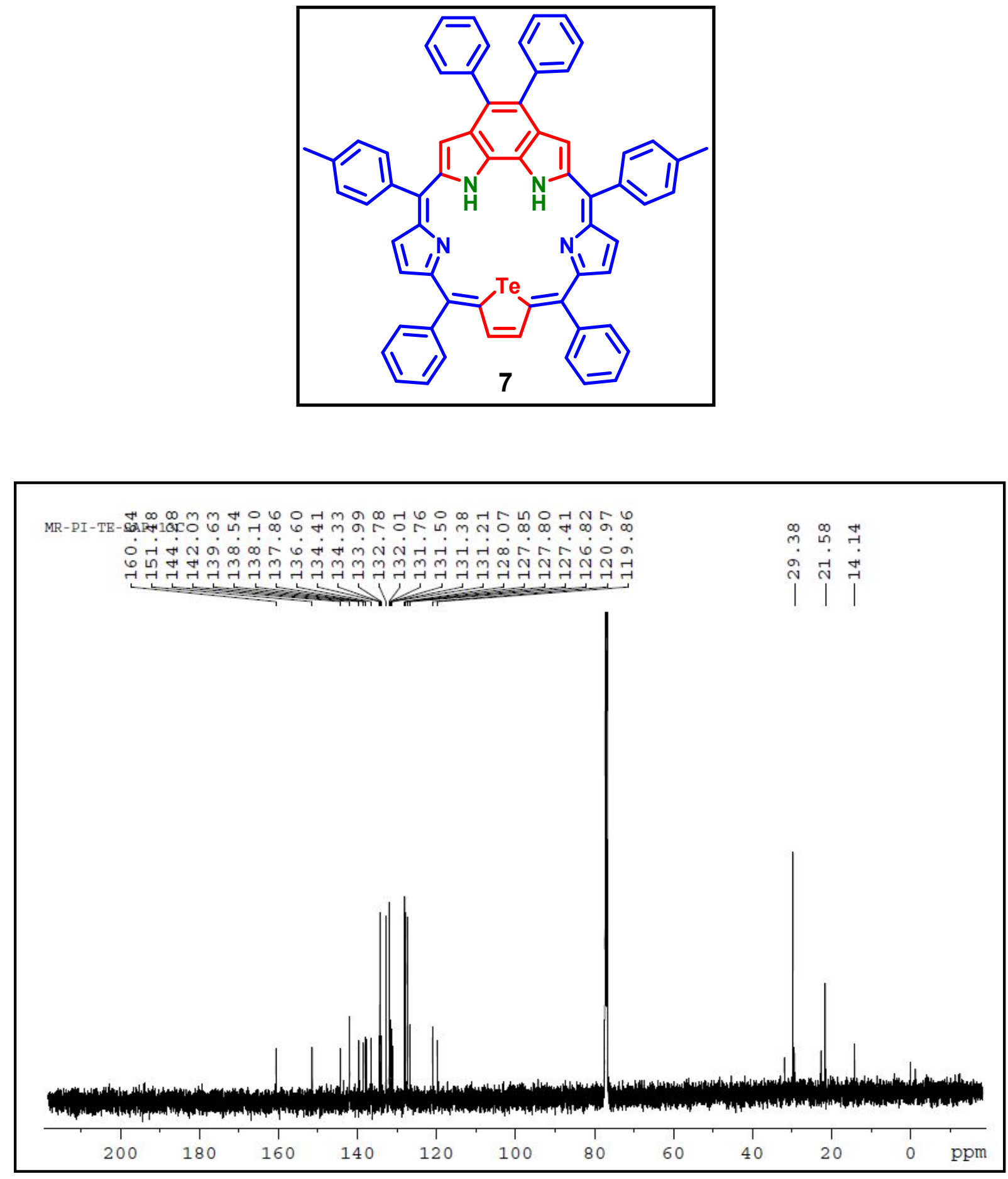

Figure S24. ${ }^{13} \mathrm{C}\left\{{ }^{1} \mathrm{H}\right\}$ NMR of compound 7 recorded in $\mathrm{CDCl}_{3}$ at $25{ }^{\circ} \mathrm{C}(125 \mathrm{MHz})$.. 
Table S1. Table of free thermal energy in normal and inverted conformation of 2a and 4-7.

\begin{tabular}{|c|c|c|c|}
\hline Compounds & $\begin{array}{l}\text { Free Thermal } \\
\text { Energy (a. u.) }\end{array}$ & $\begin{array}{c}\text { Free Thermal } \\
\text { Energy difference } \\
\text { (Inverted-Normal) } \\
\text { (a. u.) }\end{array}$ & $\begin{array}{c}\text { Free Thermal } \\
\text { Energy difference } \\
\text { (Inverted-Normal) } \\
(\mathrm{kcal} / \mathrm{mol})\end{array}$ \\
\hline Normal Benzosapphyrin & -2817.530400 & \multirow[t]{2}{*}{-0.016162} & \multirow[t]{2}{*}{-10.14181028} \\
\hline InvertedBenzosapphyrin & -2817.546562 & & \\
\hline Normal Oxabenzosapphyrin & -2837.386498 & \multirow[t]{2}{*}{-0.021581} & \multirow[t]{2}{*}{-13.54228485} \\
\hline InvertedOxabenzosapphyrin & -2837.408079 & & \\
\hline Normal Thiabenzosaphyrin & -3160.389176 & \multirow[t]{2}{*}{0.000922} & \multirow[t]{2}{*}{0.5785638586} \\
\hline Inverted Thiabenzosaphyrin & -3160.308314 & & \\
\hline Normal Selenabenzosapphyrin & -5161.592034 & \multirow[t]{2}{*}{0.007067} & \multirow[t]{2}{*}{4.4346104} \\
\hline InvertedSelenabenzosapphyrin & -5161.584967 & & \\
\hline Normal Tellurabenzosapphyrin & -2691.595374 & \multirow[t]{2}{*}{0.013304} & \multirow[t]{2}{*}{8.348387825} \\
\hline InvertedTellurabenzosapphyrin & -2691.582070 & & \\
\hline
\end{tabular}


Figure S25: Cartesian coordinates of the S0 optimized structures of the compounds inverted benzosaphyrin (4). \# Sum of imaginary frequencies $=0$;

\# Thermal free Energy $($ hartree $)=\mathbf{- 2 8 1 7 . 5 4 6 5 6 2}$.

\begin{tabular}{|c|c|c|c|}
\hline Atoms & $x$ & $Y$ & Z \\
\hline $\mathrm{N}$ & -0.82782200 & 1.64534900 & -0.15040600 \\
\hline $\mathrm{H}$ & 0.18816400 & 1.53921200 & -0.18949900 \\
\hline $\mathrm{N}$ & 1.53374800 & 2.98596100 & -0.29269600 \\
\hline $\mathrm{N}$ & -0.85440300 & -1.64743900 & -0.11239700 \\
\hline $\mathrm{H}$ & 0.16163900 & -1.55936000 & -0.18230300 \\
\hline $\mathrm{N}$ & 1.46942400 & -3.02446400 & -0.39129900 \\
\hline C & -1.35019600 & 5.41953700 & -0.10290600 \\
\hline C & 0.78676900 & 4.13682700 & -0.26153400 \\
\hline C & 2.82549500 & 3.35967400 & -0.40526500 \\
\hline C & 5.26487300 & 2.74982400 & -0.09805000 \\
\hline C & 3.56391500 & 1.11106300 & -0.94189000 \\
\hline C & 2.41502500 & -0.69573200 & -1.71223100 \\
\hline $\mathrm{H}$ & 1.71216400 & -1.34356800 & -2.21123700 \\
\hline C & 1.66345100 & 5.29742900 & -0.36482000 \\
\hline $\mathrm{H}$ & 1.34871700 & 6.33133400 & -0.40234600 \\
\hline C & 7.92042600 & 3.41234000 & 0.69979500 \\
\hline C & -0.61303900 & 4.12635800 & -0.15839200 \\
\hline C & 5.51030200 & 3.58379100 & 1.01035300 \\
\hline $\mathrm{H}$ & 4.66994300 & 3.96637300 & 1.58118300 \\
\hline C & 2.42771200 & 0.67257800 & -1.69817100 \\
\hline $\mathrm{H}$ & 1.73840200 & 1.34247500 & -2.18732800 \\
\hline C & -1.37580400 & 2.92068400 & -0.10258100 \\
\hline C & -1.82476300 & 0.70703200 & -0.05105000 \\
\hline C & 5.20635200 & -2.82674100 & -0.10009800 \\
\hline C & 6.38589100 & 2.25533600 & -0.79592100 \\
\hline $\mathrm{H}$ & 6.23321900 & 1.63342000 & -1.67353600 \\
\hline C & 6.80890000 & 3.90902900 & 1.39313500 \\
\hline $\mathrm{H}$ & 6.96240700 & 4.55451000 & 2.25537700 \\
\hline C & -3.07342900 & 1.40485500 & 0.06192600 \\
\hline C & 1.58456600 & -5.33235200 & -0.24672200 \\
\hline $\mathrm{H}$ & 1.26631100 & -6.36164300 & -0.15505600 \\
\hline C & 0.71362500 & -4.16333600 & -0.27887000 \\
\hline C & -1.08519200 & 6.35836100 & 0.90641200 \\
\hline $\mathrm{H}$ & -0.34937700 & 6.12258700 & 1.66966000 \\
\hline C & 2.93553000 & 4.81353100 & -0.42863900 \\
\hline $\mathrm{H}$ & 3.85270300 & 5.37670600 & -0.54053700 \\
\hline C & -2.32579500 & 5.74487500 & -1.06151900 \\
\hline $\mathrm{H}$ & -2.54253700 & 5.04057800 & -1.85935400 \\
\hline C & 6.33593200 & -2.38509800 & -0.81782200 \\
\hline $\mathrm{H}$ & 6.19407400 & -1.80157300 & -1.72328200 \\
\hline C & -1.83787100 & -0.69189300 & -0.05914800 \\
\hline C & 3.89016400 & 2.40778500 & -0.51533200 \\
\hline C & 5.43550600 & -3.60984000 & 1.04804700 \\
\hline $\mathrm{H}$ & 4.58801200 & -3.94963200 & 1.63523700 \\
\hline C & 7.68058900 & 2.58088500 & -0.40346300 \\
\hline $\mathrm{H}$ & 8.52258900 & 2.19331500 & -0.97324900 \\
\hline C & -2.77243500 & 2.77364600 & 0.03182000 \\
\hline $\mathrm{H}$ & -3.47766100 & 3.58690600 & 0.09953900 \\
\hline C & 3.83896400 & -2.47402800 & -0.53942600 \\
\hline C & -5.61058800 & -1.46791600 & 0.19846200 \\
\hline
\end{tabular}

\begin{tabular}{|c|c|c|c|}
\hline Atoms & $x$ & Y & Z \\
\hline $\mathrm{C}$ & 3.54117700 & -1.16946700 & -0.96243700 \\
\hline C & -1.76857900 & 7.57312300 & 0.95329500 \\
\hline $\mathrm{H}$ & -1.54952500 & 8.27680000 & 1.75347100 \\
\hline C & 2.76216900 & -3.41118000 & -0.43294200 \\
\hline C & 2.86059100 & -4.86305300 & -0.33510600 \\
\hline $\mathrm{H}$ & 3.77564000 & -5.43970500 & -0.36122700 \\
\hline C & -1.43627400 & -5.41109800 & -0.03009500 \\
\hline C & -1.42487400 & -2.91284000 & -0.07637500 \\
\hline C & -0.68247900 & -4.13108900 & -0.13277300 \\
\hline C & 7.62497200 & -2.71460600 & -0.40833400 \\
\hline $\mathrm{H}$ & 8.47424200 & -2.36917400 & -0.99413300 \\
\hline C & -5.58176400 & 1.55073400 & 0.21592800 \\
\hline C & -2.82341900 & -2.74183000 & -0.00249400 \\
\hline $\mathrm{H}$ & -3.54444600 & -3.54341600 & 0.02969800 \\
\hline C & -4.34318700 & 0.72115500 & 0.14387200 \\
\hline C & -3.00263800 & 6.96057700 & -1.01033900 \\
\hline $\mathrm{H}$ & -3.74891700 & 7.18642100 & -1.76915500 \\
\hline C & -7.72059700 & 2.24877800 & -0.71208500 \\
\hline $\mathrm{H}$ & -8.47752500 & 2.15838300 & -1.48701500 \\
\hline C & -4.35821500 & -0.66156000 & 0.10458400 \\
\hline C & -2.73683900 & 7.89928100 & -0.00345100 \\
\hline C & -3.10189600 & -1.36789300 & 0.00998700 \\
\hline C & -6.57669500 & 1.45383600 & -0.76992500 \\
\hline $\mathrm{H}$ & -6.44753800 & 0.74954200 & -1.58608800 \\
\hline C & -5.76579700 & 2.47387700 & 1.25878400 \\
\hline $\mathrm{H}$ & -5.00725100 & 2.55969100 & 2.03214700 \\
\hline C & 7.84873800 & -3.49654800 & 0.73311000 \\
\hline C & -1.35490200 & -6.37692500 & -1.04655200 \\
\hline $\mathrm{H}$ & -0.75182600 & -6.17144900 & -1.92625900 \\
\hline C & 6.72772300 & -3.93905600 & 1.44829100 \\
\hline $\mathrm{H}$ & 6.86889400 & -4.54467000 & 2.34107800 \\
\hline c & -2.05453300 & -7.57878500 & -0.95163300 \\
\hline $\mathrm{H}$ & -1.98103300 & -8.30286400 & -1.76027900 \\
\hline C & 9.32770300 & 3.73901800 & 1.13932900 \\
\hline $\mathrm{H}$ & 9.37181700 & 4.70009400 & 1.66241700 \\
\hline H & 10.01377800 & 3.78555200 & 0.28657500 \\
\hline $\mathrm{H}$ & 9.71781500 & 2.97650600 & 1.82731900 \\
\hline C & -5.95032900 & -2.37953600 & -0.81509400 \\
\hline $\mathrm{H}$ & -5.30290400 & -2.47494200 & -1.68258300 \\
\hline C & -2.24581400 & -5.69741100 & 1.08234500 \\
\hline $\mathrm{H}$ & -2.31911500 & -4.97197500 & 1.88729900 \\
\hline C & -6.46417300 & -1.35774900 & 1.30749400 \\
\hline $\mathrm{H}$ & -6.21339100 & -0.66156800 & 2.10204800 \\
\hline C & -2.85745600 & -7.86632600 & 0.15900300 \\
\hline C & -6.91152800 & 3.26821300 & 1.31852300 \\
\hline $\mathrm{H}$ & -7.03663200 & 3.97111900 & 2.13826300 \\
\hline C & -7.11052500 & -3.14954600 & -0.72689800 \\
\hline $\mathrm{H}$ & -7.35775000 & -3.84367300 & -1.52613300 \\
\hline C & -7.89325500 & 3.15915500 & 0.33235500 \\
\hline $\mathrm{H}$ & -8.78530100 & 3.77846100 & 0.37712200 \\
\hline
\end{tabular}




\begin{tabular}{cccc}
\hline Atoms & $\mathrm{X}$ & $\mathrm{Y}$ & $\mathrm{Z}$ \\
\hline $\mathrm{C}$ & -2.94054100 & -6.90135100 & 1.17194200 \\
$\mathrm{H}$ & -3.55652800 & -7.09606600 & 2.04730600 \\
$\mathrm{C}$ & -7.95114900 & -3.02730100 & 0.38063700 \\
$\mathrm{H}$ & -8.85452200 & -3.62747500 & 0.45122800 \\
$\mathrm{C}$ & 9.24890100 & -3.82843300 & 1.19138000 \\
$\mathrm{H}$ & 9.95135300 & -3.84937100 & 0.35136900 \\
H & 9.28781800 & -4.80293200 & 1.68994700 \\
$\mathrm{H}$ & 9.62112400 & -3.08335200 & 1.90777000 \\
$\mathrm{C}$ & -7.62238500 & -2.12880100 & 1.39758900 \\
H & -8.26830300 & -2.02839100 & 2.26609400 \\
\hline
\end{tabular}

\begin{tabular}{cccc}
\hline Atoms & $\mathrm{X}$ & $\mathrm{Y}$ & $\mathrm{Z}$ \\
\hline $\mathrm{C}$ & -3.59205200 & -9.18134400 & 0.27442600 \\
$\mathrm{H}$ & -3.76858300 & -9.62933200 & -0.70904900 \\
$\mathrm{H}$ & -3.01809000 & -9.90784400 & 0.86545400 \\
$\mathrm{H}$ & -4.56166200 & -9.05758700 & 0.76916800 \\
$\mathrm{C}$ & -3.45986900 & 9.22518900 & 0.03201100 \\
$\mathrm{H}$ & -3.36041200 & 9.70968300 & 1.00878000 \\
$\mathrm{H}$ & -3.05943000 & 9.91790900 & -0.72024400 \\
$\mathrm{H}$ & -4.52879600 & 9.10550800 & -0.17856500 \\
$\mathrm{H}$ & 5.02202600 & -0.05263800 & 0.05341900 \\
$\mathrm{~N}$ & 4.26700800 & -0.03871000 & -0.61851700 \\
\hline
\end{tabular}

Figure S26: Cartesian coordinates of the S0 optimized structures of the compounds normalbenzosaphyrin. \# Sum of imaginary frequencies=0; \# Thermal Free Energy (hartree) =

\section{-2817.530400 .}

\begin{tabular}{lrrr}
\hline Atoms & $\mathrm{X}$ & $\mathrm{Y}$ & $\mathrm{Z}$ \\
\hline $\mathrm{C}$ & -4.18834800 & -1.14893600 & 0.25522200 \\
$\mathrm{C}$ & -5.42336500 & -0.68528800 & 0.82919400 \\
$\mathrm{C}$ & -5.42146000 & 0.67275200 & 0.84740000 \\
$\mathrm{C}$ & -4.18636900 & 1.14792900 & 0.28288200 \\
$\mathrm{C}$ & -0.69949300 & 3.93627000 & -0.45657300 \\
$\mathrm{C}$ & -1.54361000 & 5.05584600 & -0.83433700 \\
$\mathrm{C}$ & -2.82196500 & 4.61889800 & -0.73794700 \\
$\mathrm{C}$ & -2.75903000 & 3.22411300 & -0.30088600 \\
$\mathrm{~N}$ & -1.47207400 & 2.81748600 & -0.18328000 \\
$\mathrm{C}$ & -3.94996800 & 2.50890500 & 0.03614000 \\
$\mathrm{C}$ & 1.96727200 & 0.69238500 & 0.12733300 \\
$\mathrm{C}$ & 3.21172500 & 1.39254600 & 0.20935000 \\
$\mathrm{C}$ & 2.90104700 & 2.75541400 & 0.07686900 \\
$\mathrm{C}$ & 1.50502300 & 2.86761400 & -0.10958500 \\
$\mathrm{~N}$ & 0.96459800 & 1.59840200 & -0.05403700 \\
$\mathrm{C}$ & 1.96302500 & -0.69259600 & 0.15436800 \\
$\mathrm{C}$ & 3.20343200 & -1.39460000 & 0.26621400 \\
$\mathrm{C}$ & 2.89565800 & -2.75634900 & 0.11760500 \\
$\mathrm{C}$ & 1.50164500 & -2.86835800 & -0.08321200 \\
$\mathrm{~N}$ & 0.96108300 & -1.59897500 & -0.03135400 \\
$\mathrm{C}$ & -0.69753600 & -3.93196900 & -0.47480700 \\
$\mathrm{C}$ & -1.53660300 & -5.04491600 & -0.88244400 \\
$\mathrm{C}$ & -2.81568300 & -4.60591600 & -0.80616900 \\
$\mathrm{C}$ & -2.75823900 & -3.21726800 & -0.34915600 \\
$\mathrm{~N}$ & -1.47284500 & -2.81507200 & -0.20257200 \\
$\mathrm{C}$ & -3.95258300 & -2.50512600 & -0.01844000 \\
$\mathrm{C}$ & 0.69927000 & 4.00824100 & -0.35633500 \\
$\mathrm{C}$ & 0.69927700 & -4.00708300 & -0.35038700 \\
$\mathrm{C}$ & 4.46107200 & -0.69511100 & 0.41440700 \\
$\mathrm{C}$ & 4.46523400 & 0.69113500 & 0.38153100 \\
$\mathrm{C}$ & 1.38801100 & 5.31906400 & -0.51610300 \\
$\mathrm{C}$ & 1.38834800 & -5.31770800 & -0.50950600 \\
$\mathrm{C}$ & 5.70480600 & 1.50589500 & 0.54586500 \\
$\mathrm{C}$ & 5.70085600 & -1.51570000 & 0.53696200 \\
$\mathrm{C}$ & -5.17190600 & 3.37748400 & 0.18724200 \\
\hline & & & \\
\hline
\end{tabular}

\begin{tabular}{|c|c|c|c|}
\hline Atoms & $x$ & $Y$ & Z \\
\hline$C$ & -5.18024700 & -3.37091500 & 0.09716100 \\
\hline C & -5.26675200 & 4.31565500 & 1.22669500 \\
\hline C & -6.39719000 & 5.11828100 & 1.36703700 \\
\hline C & -7.46914500 & 5.02547900 & 0.46874000 \\
\hline C & -7.37289900 & 4.09092700 & -0.56968400 \\
\hline C & -6.24737400 & 3.27911700 & -0.70746800 \\
\hline C & -6.24138800 & -3.24776900 & -0.81139600 \\
\hline C & -7.37087600 & -4.05975700 & -0.71126500 \\
\hline C & -7.48757900 & -5.01602000 & 0.30489000 \\
\hline C & -6.42700500 & -5.13829500 & 1.21329300 \\
\hline C & -5.29291800 & -4.33536200 & 1.11065400 \\
\hline C & 2.37309500 & 5.50550200 & -1.50077400 \\
\hline C & 3.01328400 & 6.73314200 & -1.65024500 \\
\hline C & 2.70508400 & 7.81933600 & -0.82022100 \\
\hline C & 1.72394000 & 7.63296600 & 0.16140400 \\
\hline C & 1.07625700 & 6.40846800 & 0.31315200 \\
\hline C & 1.05632800 & -6.41429200 & 0.30318900 \\
\hline C & 1.70898100 & -7.63665400 & 0.15911100 \\
\hline C & 2.71286100 & -7.81562600 & -0.80136200 \\
\hline C & 3.04555600 & -6.72112900 & -1.61002700 \\
\hline C & 2.39968900 & -5.49511300 & -1.46849600 \\
\hline C & 6.76188600 & -1.37492200 & -0.37180100 \\
\hline C & 7.90491700 & -2.16521500 & -0.26390600 \\
\hline C & 8.01123200 & -3.11457200 & 0.75411000 \\
\hline C & 6.96331800 & -3.26829400 & 1.66271400 \\
\hline C & 5.81821500 & -2.47922200 & 1.55279700 \\
\hline C & 6.10329400 & 2.41497000 & -0.44796800 \\
\hline C & 7.24977200 & 3.19363000 & -0.28777900 \\
\hline C & 8.01681100 & 3.08277500 & 0.87261700 \\
\hline C & 7.62913300 & 2.18627500 & 1.87003800 \\
\hline C & 6.48517000 & 1.40644400 & 1.70842700 \\
\hline C & -8.67595000 & 5.92353600 & 0.60271300 \\
\hline C & -8.72468600 & -5.87243400 & 0.43472400 \\
\hline C & 3.42614300 & 9.13806000 & -0.96461800 \\
\hline C & 3.39581700 & -9.15083600 & -0.97686300 \\
\hline
\end{tabular}




\begin{tabular}{cccc}
\hline Atoms & $\mathrm{X}$ & $\mathrm{Y}$ & $\mathrm{Z}$ \\
\hline $\mathrm{H}$ & 8.90247900 & -3.72976300 & 0.83774300 \\
$\mathrm{H}$ & 7.03626400 & -4.00200500 & 2.46057100 \\
$\mathrm{H}$ & 8.71298800 & -2.04116800 & -0.97928900 \\
$\mathrm{H}$ & 6.68453800 & -0.64153600 & -1.16762600 \\
$\mathrm{H}$ & 5.00781600 & -2.59992600 & 2.26556100 \\
$\mathrm{H}$ & 8.21741800 & 2.09485200 & 2.77866300 \\
$\mathrm{H}$ & 6.18741100 & 0.71217600 & 2.48752300 \\
$\mathrm{H}$ & 8.90849000 & 3.68990500 & 0.99905400 \\
$\mathrm{H}$ & 7.54458800 & 3.88509500 & -1.07220000 \\
$\mathrm{H}$ & 5.51478800 & 2.49986400 & -1.35676800 \\
$\mathrm{H}$ & 3.58851700 & -3.58240400 & 0.13333800 \\
$\mathrm{H}$ & 3.59035300 & 3.58418800 & 0.10278900 \\
$\mathrm{H}$ & 2.61911900 & 4.68460000 & -2.16704300 \\
$\mathrm{H}$ & 3.76044900 & 6.85228000 & -2.43122700 \\
$\mathrm{H}$ & 1.46474200 & 8.45728900 & 0.82125100 \\
$\mathrm{H}$ & 0.32616400 & 6.28631700 & 1.08809800 \\
$\mathrm{H}$ & 4.37383400 & 9.13424000 & -0.41154100 \\
$\mathrm{H}$ & 2.82666600 & 9.96680700 & -0.57699100 \\
$\mathrm{H}$ & -9.56040700 & 5.47843800 & 0.13771400 \\
$\mathrm{H}$ & -8.91016300 & 6.12602700 & 1.65240100 \\
$\mathrm{H}$ & -6.44915300 & 5.82780600 & 2.18941600 \\
$\mathrm{H}$ & -4.44802300 & 4.40621300 & 1.93419300 \\
$\mathrm{H}$ & -6.19663200 & 2.56005000 & -1.51968900 \\
$\mathrm{H}$ & -8.18975800 & 3.99654600 & -1.28106300 \\
$\mathrm{H}$ & -1.19703000 & 6.02985600 & -1.14601700 \\
\hline & & &
\end{tabular}

\begin{tabular}{crrr}
\hline Atoms & $\mathrm{X}$ & $\mathrm{Y}$ & $\mathrm{Z}$ \\
\hline $\mathrm{H}$ & -3.72379100 & 5.17039900 & -0.95504600 \\
$\mathrm{H}$ & -6.19261400 & 1.31675600 & 1.23728200 \\
$\mathrm{H}$ & -6.19608300 & -1.33743900 & 1.20214100 \\
$\mathrm{H}$ & -6.17306000 & -2.51448500 & -1.60953000 \\
$\mathrm{H}$ & -4.48174700 & -4.45156800 & 1.82307400 \\
$\mathrm{H}$ & -6.48959100 & -5.87367000 & 2.01192900 \\
$\mathrm{H}$ & -8.17325600 & -3.95031700 & -1.43686900 \\
$\mathrm{H}$ & -9.42347100 & -5.45198500 & 1.16886300 \\
$\mathrm{H}$ & -8.47808500 & -6.88520900 & 0.76840300 \\
$\mathrm{H}$ & -3.71423400 & -5.15235100 & -1.04827400 \\
$\mathrm{H}$ & -1.18612100 & -6.01581500 & -1.19944400 \\
$\mathrm{H}$ & 2.66990800 & -4.66651300 & -2.11542600 \\
$\mathrm{H}$ & 0.29167500 & -6.29644700 & 1.06449400 \\
$\mathrm{H}$ & 1.43838100 & -8.46420200 & 0.81038400 \\
$\mathrm{H}$ & 3.81998100 & -6.83012000 & -2.36529000 \\
$\mathrm{H}$ & 3.43788000 & -9.70618600 & -0.03515000 \\
$\mathrm{H}$ & -0.05243600 & 1.48880000 & -0.06470600 \\
$\mathrm{H}$ & -0.05523200 & -1.48768600 & -0.06435300 \\
$\mathrm{H}$ & -8.50513600 & 6.89256100 & 0.11707400 \\
$\mathrm{H}$ & -9.25916400 & -5.95009400 & -0.51639800 \\
$\mathrm{H}$ & 3.66420000 & 9.35192800 & -2.01117700 \\
$\mathrm{H}$ & 2.85986500 & -9.77838200 & -1.70014200 \\
$\mathrm{H}$ & 4.41863600 & -9.03203400 & -1.34655600 \\
$\mathrm{~N}$ & -3.43824700 & 0.00198000 & 0.00536500 \\
$\mathrm{H}$ & -2.54746300 & 0.00734000 & -0.46674000 \\
\hline & & &
\end{tabular}

Figure S27: Cartesian coordinates of the S0 optimized structures of the compounds normaloxabenzosaphyrin. \#Sum of imaginary frequencies=0; \#Thermal Free Energy (hartree) $=\mathbf{- 2 8 3 7 . 3 8 6 4 9 8}$.

\begin{tabular}{crrr}
\hline Atoms & $\mathrm{X}$ & $\mathrm{Y}$ & $\mathrm{Z}$ \\
\hline $\mathrm{C}$ & -4.19384300 & -1.11690000 & 0.29126400 \\
$\mathrm{C}$ & -5.38231700 & -0.67617500 & 0.94781400 \\
$\mathrm{C}$ & -5.37742500 & 0.68227000 & 0.96343700 \\
$\mathrm{C}$ & -4.18701300 & 1.12932500 & 0.31437100 \\
$\mathrm{C}$ & -0.69810400 & 3.91782400 & -0.55195500 \\
$\mathrm{C}$ & -1.55515000 & 4.99326500 & -1.01891700 \\
$\mathrm{C}$ & -2.82623400 & 4.53273800 & -0.91812700 \\
$\mathrm{C}$ & -2.73975100 & 3.17083100 & -0.38621200 \\
$\mathrm{~N}$ & -1.45477100 & 2.80534600 & -0.21785000 \\
$\mathrm{C}$ & -3.92460600 & 2.46412800 & 0.01547800 \\
$\mathrm{C}$ & 1.94802200 & 0.69009900 & 0.13742200 \\
$\mathrm{C}$ & 3.19052500 & 1.38736200 & 0.24926800 \\
$\mathrm{C}$ & 2.88394300 & 2.75025600 & 0.09485500 \\
$\mathrm{C}$ & 1.49425300 & 2.85954800 & -0.13281400 \\
$\mathrm{~N}$ & 0.95145600 & 1.59138200 & -0.07794000 \\
$\mathrm{C}$ & 1.94039600 & -0.69705200 & 0.16303400 \\
$\mathrm{C}$ & 3.17534700 & -1.40121000 & 0.30349400 \\
$\mathrm{C}$ & 2.86712000 & -2.76141500 & 0.13034100 \\
$\mathrm{C}$ & 1.47950200 & -2.86492800 & -0.11182000 \\
$\mathrm{~N}$ & 0.94141400 & -1.59454500 & -0.05905300 \\
$\mathrm{C}$ & -0.71078700 & -3.90981800 & -0.57230200 \\
$\mathrm{C}$ & -1.56613000 & -4.97480800 & -1.06585400 \\
\hline & & &
\end{tabular}

\begin{tabular}{cccc}
\hline Atoms & $\mathrm{X}$ & $\mathrm{Y}$ & $\mathrm{Z}$ \\
\hline $\mathrm{C}$ & -2.83632600 & -4.50878900 & -0.97891900 \\
C & -2.75101600 & -3.15448200 & -0.42801800 \\
N & -1.46682600 & -2.79769900 & -0.23619700 \\
C & -3.93684800 & -2.44770300 & -0.02998200 \\
C & 0.69644700 & 3.99792500 & -0.42911700 \\
C & 0.68152300 & -3.99796700 & -0.42889600 \\
C & 4.42921100 & -0.70380600 & 0.49109600 \\
C & 4.43680400 & 0.68295800 & 0.45975800 \\
C & 1.38507900 & 5.30158800 & -0.62526500 \\
C & 1.36652700 & -5.30295700 & -0.62786700 \\
C & 5.67170900 & 1.49564600 & 0.66466600 \\
C & 5.66288200 & -1.52672100 & 0.65240400 \\
C & -5.12368000 & 3.35757300 & 0.21040200 \\
C & -5.14412800 & -3.33552700 & 0.13703600 \\
C & -5.13288100 & 4.34177300 & 1.21068500 \\
C & -6.23934100 & 5.16941100 & 1.39065400 \\
C & -7.37025800 & 5.05768100 & 0.57029800 \\
C & -7.35825700 & 4.07838700 & -0.43105900 \\
C & -6.25766800 & 3.24050600 & -0.60690000 \\
C & -6.26731100 & -3.19638800 & -0.69118500 \\
C & -7.37447500 & -4.03180500 & -0.54489600 \\
C & -7.40580500 & -5.02687400 & 0.43986800 \\
\hline & & &
\end{tabular}




\begin{tabular}{|c|c|c|c|}
\hline Atoms & $x$ & Y & Z \\
\hline $\mathrm{C}$ & -6.28225200 & -5.16561700 & 1.26685300 \\
\hline C & -5.16973200 & -4.34082400 & 1.11634400 \\
\hline C & 2.41876200 & 5.44473000 & -1.56650100 \\
\hline C & 3.05498000 & 6.66982900 & -1.75030400 \\
\hline $\mathrm{C}$ & 2.69499900 & 7.79596300 & -0.99842100 \\
\hline C & 1.66460500 & 7.65300500 & -0.06036100 \\
\hline C & 1.01979500 & 6.43216100 & 0.12440800 \\
\hline C & 0.97986700 & -6.43979800 & 0.10030800 \\
\hline C & 1.62562000 & -7.66135700 & -0.08193500 \\
\hline C & 2.67491900 & -7.79864300 & -0.99849500 \\
\hline C & 3.06137900 & -6.66351700 & -1.72474800 \\
\hline $\mathrm{C}$ & 2.42447100 & -5.43918100 & -1.54414000 \\
\hline C & 6.75752400 & -1.38141200 & -0.21499800 \\
\hline C & 7.89514400 & -2.17341200 & -0.06901900 \\
\hline C & 7.96251500 & -3.12934100 & 0.94620200 \\
\hline C & 6.88079800 & -3.28823000 & 1.81335800 \\
\hline C & 5.74131300 & -2.49732000 & 1.66538600 \\
\hline C & 6.10358300 & 2.40586700 & -0.31408000 \\
\hline C & 7.24437400 & 3.18391800 & -0.11466700 \\
\hline C & 7.97227500 & 3.07132100 & 1.07049300 \\
\hline C & 7.55131700 & 2.17345200 & 2.05310700 \\
\hline C & 6.41306500 & 1.39439000 & 1.85239500 \\
\hline $\mathrm{C}$ & -8.55139100 & 5.98271600 & 0.74308900 \\
\hline $\mathrm{C}$ & -8.61836600 & -5.90819800 & 0.62224800 \\
\hline $\mathrm{C}$ & 3.41160200 & 9.11298000 & -1.17555100 \\
\hline $\mathrm{C}$ & 3.35563100 & -9.12847300 & -1.21739100 \\
\hline $\mathrm{H}$ & 8.84967700 & -3.74571900 & 1.05943500 \\
\hline $\mathrm{H}$ & 6.92304800 & -4.02719500 & 2.60859700 \\
\hline $\mathrm{H}$ & 8.72966300 & -2.04557400 & -0.75268300 \\
\hline $\mathrm{H}$ & 6.71079100 & -0.64300100 & -1.00849700 \\
\hline $\mathrm{H}$ & 4.90465700 & -2.62160000 & 2.34646900 \\
\hline $\mathrm{H}$ & 8.10904000 & 2.08047800 & 2.98067100 \\
\hline $\mathrm{H}$ & 6.08918800 & 0.69925600 & 2.62022800 \\
\hline $\mathrm{H}$ & 8.85938300 & 3.67805000 & 1.22740900 \\
\hline $\mathrm{H}$ & 7.56543500 & 3.87627700 & -0.88794100 \\
\hline $\mathrm{H}$ & 5.54599100 & 2.49191900 & -1.24208200 \\
\hline $\mathrm{H}$ & 3.55636800 & -3.59031400 & 0.15714800 \\
\hline $\mathrm{H}$ & 3.57234700 & 3.57938800 & 0.13285200 \\
\hline
\end{tabular}

\begin{tabular}{crrr}
\hline Atoms & $\mathrm{X}$ & $\mathrm{Y}$ & $\mathrm{Z}$ \\
\hline $\mathrm{H}$ & 2.70522400 & 4.59205700 & -2.17384500 \\
$\mathrm{H}$ & 3.84045400 & 6.75487700 & -2.49728400 \\
$\mathrm{H}$ & 1.36415300 & 8.50942100 & 0.53832600 \\
$\mathrm{H}$ & 0.23139100 & 6.34363600 & 0.86492600 \\
$\mathrm{H}$ & 4.28259500 & 9.18232000 & -0.51169300 \\
$\mathrm{H}$ & 2.75841100 & 9.95911700 & -0.94251300 \\
$\mathrm{H}$ & -9.48185600 & 5.51144600 & 0.41288500 \\
$\mathrm{H}$ & -8.67514500 & 6.28312600 & 1.78792900 \\
$\mathrm{H}$ & -6.22563400 & 5.91447000 & 2.18247400 \\
$\mathrm{H}$ & -4.26653700 & 4.44844100 & 1.85640400 \\
$\mathrm{H}$ & -6.27136400 & 2.48731700 & -1.38910500 \\
$\mathrm{H}$ & -8.22174300 & 3.96964100 & -1.08283800 \\
$\mathrm{H}$ & -1.22278400 & 5.95057200 & -1.39252300 \\
$\mathrm{H}$ & -3.73632500 & 5.04642200 & -1.18897800 \\
$\mathrm{H}$ & -6.11536700 & 1.33413600 & 1.40163800 \\
$\mathrm{H}$ & -6.12472100 & -1.33275400 & 1.37120300 \\
$\mathrm{H}$ & -6.26464800 & -2.43323600 & -1.46381500 \\
$\mathrm{H}$ & -4.30800000 & -4.47078500 & 1.76393000 \\
$\mathrm{H}$ & -6.27802500 & -5.93209200 & 2.03816900 \\
$\mathrm{H}$ & -8.22651700 & -3.91049600 & -1.20938600 \\
$\mathrm{H}$ & -9.27353800 & -5.52281600 & 1.41363900 \\
$\mathrm{H}$ & -8.33498200 & -6.92644300 & 0.90624600 \\
$\mathrm{H}$ & -3.74479200 & -5.01416300 & -1.27020500 \\
$\mathrm{H}$ & -1.23297700 & -5.92901000 & -1.44659200 \\
$\mathrm{H}$ & 2.73537200 & -4.57906500 & -2.12833400 \\
$\mathrm{H}$ & 0.17894900 & -6.35513200 & 0.82773700 \\
$\mathrm{H}$ & 1.31238700 & -8.52161700 & 0.50440000 \\
$\mathrm{H}$ & 3.87134100 & -6.74010000 & -2.44607200 \\
$\mathrm{H}$ & 3.17639400 & -9.81201200 & -0.38275200 \\
$\mathrm{H}$ & -0.06699700 & 1.47586000 & -0.11333800 \\
$\mathrm{H}$ & -0.07539800 & -1.47336600 & -0.11342200 \\
$\mathrm{H}$ & -8.42533400 & 6.90040500 & 0.15485000 \\
$\mathrm{H}$ & -9.21329800 & -5.96517100 & -0.29384200 \\
$\mathrm{H}$ & 3.77484300 & 9.23658200 & -2.20018100 \\
$\mathrm{H}$ & 2.98605400 & -9.61757500 & -2.12729300 \\
\hline & 4.43783500 & -9.01043700 & -1.33276900 \\
\hline & -3.43803900 & 0.00730100 & -0.02862800 \\
\hline & & & \\
\hline & & & \\
& & &
\end{tabular}

Figure S28: Cartesian coordinates of the S0 optimized structures of the compounds invertedselenabenzosaphyrin. \#Sum of imaginary frequencies $=0$; \#Thermal free Energy $($ hartree $)=\mathbf{- 5 1 6 1 . 5 8 4 9 6 7}$.

\begin{tabular}{lrrc}
\hline Atoms & $\mathrm{X}$ & $\mathrm{Y}$ & $\mathrm{Z}$ \\
\hline $\mathrm{N}$ & -1.06331200 & 1.65796400 & -0.08564800 \\
$\mathrm{H}$ & -0.04792400 & 1.56129000 & -0.09895200 \\
$\mathrm{~N}$ & 1.29977200 & 3.08484800 & -0.22655800 \\
$\mathrm{~N}$ & -1.09243500 & -1.65688600 & -0.05330100 \\
$\mathrm{H}$ & -0.07610100 & -1.57829200 & -0.09093200 \\
$\mathrm{~N}$ & 1.23334200 & -3.11652400 & -0.32382800 \\
$\mathrm{C}$ & -1.67083600 & 5.42102300 & -0.16053200 \\
$\mathrm{C}$ & 0.50150400 & 4.20439500 & -0.26039300 \\
\hline
\end{tabular}

\begin{tabular}{lcrc}
\hline Atoms & $\mathrm{X}$ & $\mathrm{Y}$ & $\mathrm{Z}$ \\
\hline $\mathrm{C}$ & 2.56966100 & 3.51721700 & -0.35388100 \\
$\mathrm{C}$ & 5.04537100 & 3.19185200 & -0.02193800 \\
$\mathrm{C}$ & 3.55536100 & 1.29196500 & -0.67391100 \\
$\mathrm{C}$ & 2.41899000 & -0.69794000 & -1.30908400 \\
$\mathrm{H}$ & 1.62437200 & -1.27944900 & -1.75566100 \\
$\mathrm{C}$ & 1.31965000 & 5.39750100 & -0.42966200 \\
$\mathrm{H}$ & 0.95451300 & 6.41002600 & -0.52339100 \\
$\mathrm{C}$ & 7.60318100 & 4.24238500 & 0.64292200 \\
\hline
\end{tabular}




\begin{tabular}{|c|c|c|c|}
\hline Atoms & $x$ & $Y$ & Z \\
\hline $\mathrm{C}$ & -0.89412100 & 4.14850900 & -0.16186800 \\
\hline $\mathrm{C}$ & 5.21476700 & 4.05262100 & 1.07653900 \\
\hline $\mathrm{H}$ & 4.35519900 & 4.30886900 & 1.68742300 \\
\hline $\mathrm{C}$ & 2.43426800 & 0.66999000 & -1.29601700 \\
\hline $\mathrm{H}$ & 1.65306100 & 1.27557800 & -1.73448300 \\
\hline $\mathrm{C}$ & -1.62721600 & 2.92787700 & -0.06607900 \\
\hline $\mathrm{C}$ & -2.05522500 & 0.71059700 & 0.01685100 \\
\hline C & 4.98157900 & -3.26577900 & -0.02844300 \\
\hline C & 6.18492000 & 2.87133400 & -0.78297900 \\
\hline $\mathrm{H}$ & 6.07951800 & 2.21960100 & -1.64481100 \\
\hline C & 6.46909900 & 4.56495300 & 1.39906500 \\
\hline $\mathrm{H}$ & 6.56980500 & 5.22353600 & 2.25830600 \\
\hline $\mathrm{C}$ & -3.30957700 & 1.40230500 & 0.11149600 \\
\hline C & 1.23496800 & -5.43729000 & -0.30191400 \\
\hline $\mathrm{H}$ & 0.86486400 & -6.45154900 & -0.26519300 \\
\hline$C$ & 0.42465100 & -4.22727500 & -0.27289000 \\
\hline C & -1.45609400 & 6.39304700 & 0.82840800 \\
\hline $\mathrm{H}$ & -0.72976700 & 6.20068400 & 1.61184600 \\
\hline $\mathrm{C}$ & 2.61121800 & 4.97070000 & -0.46839800 \\
\hline $\mathrm{H}$ & 3.50193700 & 5.56667200 & -0.60810500 \\
\hline $\mathrm{C}$ & -2.63195800 & 5.69045100 & -1.14985000 \\
\hline $\mathrm{H}$ & -2.80981800 & 4.95874400 & -1.93166900 \\
\hline $\mathrm{C}$ & 6.11921300 & -3.00033100 & -0.81252800 \\
\hline $\mathrm{H}$ & 6.01627100 & -2.38912900 & -1.70395700 \\
\hline $\mathrm{C}$ & -2.06935700 & -0.69112300 & 0.00543700 \\
\hline C & 3.71219200 & 2.64258700 & -0.37699500 \\
\hline $\mathrm{C}$ & 5.14690300 & -4.07017400 & 1.11204700 \\
\hline $\mathrm{H}$ & 4.28856100 & -4.28138800 & 1.74163600 \\
\hline $\mathrm{C}$ & 7.43464000 & 3.38835700 & -0.45567300 \\
\hline $\mathrm{H}$ & 8.29405500 & 3.13295900 & -1.07077400 \\
\hline $\mathrm{C}$ & -3.02009900 & 2.77198100 & 0.06295200 \\
\hline $\mathrm{H}$ & -3.73270000 & 3.57901800 & 0.10954000 \\
\hline $\mathrm{C}$ & 3.65516500 & -2.70792000 & -0.40314900 \\
\hline $\mathrm{C}$ & -5.85003700 & -1.46314700 & 0.21938500 \\
\hline $\mathrm{C}$ & 3.52520200 & -1.35378600 & -0.69602100 \\
\hline $\mathrm{C}$ & -2.17673900 & 7.58668300 & 0.82759500 \\
\hline $\mathrm{H}$ & -1.99708000 & 8.31696900 & 1.61264200 \\
\hline $\mathrm{C}$ & 2.50249800 & -3.56737800 & -0.37851000 \\
\hline $\mathrm{C}$ & 2.53044400 & -5.02609700 & -0.36537800 \\
\hline $\mathrm{H}$ & 3.41753200 & -5.64110700 & -0.41601600 \\
\hline $\mathrm{C}$ & -1.76334100 & -5.40831200 & -0.08411200 \\
\hline $\mathrm{C}$ & -1.67926800 & -2.91589000 & -0.04681100 \\
\hline $\mathrm{C}$ & -0.96680700 & -4.14925200 & -0.13608100 \\
\hline $\mathrm{C}$ & 7.36413800 & -3.51921700 & -0.46879500 \\
\hline $\mathrm{H}$ & 8.22251600 & -3.30780900 & -1.10178700 \\
\hline $\mathrm{C}$ & -5.81805000 & 1.55598600 & 0.25128900 \\
\hline $\mathrm{C}$ & -3.07361500 & -2.73427400 & 0.01925100 \\
\hline $\mathrm{H}$ & -3.80163700 & -3.52881500 & 0.02924200 \\
\hline $\mathrm{C}$ & -4.58080500 & 0.72419400 & 0.18508000 \\
\hline $\mathrm{C}$ & -3.34488600 & 6.88617600 & -1.14709500 \\
\hline $\mathrm{H}$ & -4.07843300 & 7.06939500 & -1.92849700 \\
\hline $\mathrm{C}$ & -7.95046800 & 2.25792900 & -0.68655500 \\
\hline
\end{tabular}

\begin{tabular}{|c|c|c|c|}
\hline Atoms & $x$ & $Y$ & Z \\
\hline $\mathrm{H}$ & -8.70290500 & 2.17054400 & -1.46523400 \\
\hline C & -4.59648800 & -0.65699300 & 0.14054300 \\
\hline C & -3.13148600 & 7.85788200 & -0.15936700 \\
\hline C & -3.33947800 & -1.35930800 & 0.05261700 \\
\hline C & -6.80730600 & 1.46213500 & -0.74031200 \\
\hline $\mathrm{H}$ & -6.67439100 & 0.75984300 & -1.55678500 \\
\hline $\mathrm{C}$ & -6.00661300 & 2.47633400 & 1.29558400 \\
\hline $\mathrm{H}$ & -5.25254400 & 2.55936100 & 2.07275200 \\
\hline C & 7.52860500 & -4.31848300 & 0.67056500 \\
\hline C & -1.73859900 & -6.31941900 & -1.15164100 \\
\hline $\mathrm{H}$ & -1.14733900 & -6.08851200 & -2.03229800 \\
\hline C & 6.39626000 & -4.58432500 & 1.45107600 \\
\hline $\mathrm{H}$ & 6.49456100 & -5.19926800 & 2.34233700 \\
\hline C & -2.47787200 & -7.50003500 & -1.10214200 \\
\hline $\mathrm{H}$ & -2.44848500 & -8.18224700 & -1.94821200 \\
\hline C & 8.96666300 & 4.77366600 & 1.01371700 \\
\hline $\mathrm{H}$ & 8.89521600 & 5.73143200 & 1.53747400 \\
\hline $\mathrm{H}$ & 9.59502800 & 4.91585200 & 0.12920000 \\
\hline $\mathrm{H}$ & 9.49497800 & 4.07797000 & 1.67789200 \\
\hline C & -6.18914700 & -2.36004300 & -0.80710900 \\
\hline $\mathrm{H}$ & -5.53997700 & -2.44586400 & -1.67353100 \\
\hline C & -2.55577000 & -5.72423400 & 1.03191500 \\
\hline $\mathrm{H}$ & -2.58553800 & -5.03953600 & 1.87374600 \\
\hline C & -6.70653500 & -1.36370000 & 1.32689900 \\
\hline $\mathrm{H}$ & -6.45620400 & -0.67812900 & 2.13001200 \\
\hline C & -3.26394000 & -7.81857500 & 0.01192800 \\
\hline C & -7.15171600 & 3.27140600 & 1.35080900 \\
\hline $\mathrm{H}$ & -7.28094800 & 3.97193600 & 2.17102700 \\
\hline C & -7.35175300 & -3.12737500 & -0.73201600 \\
\hline $\mathrm{H}$ & -7.59886200 & -3.81001900 & -1.54019200 \\
\hline C & -8.12778700 & 3.16559500 & 0.35909500 \\
\hline $\mathrm{H}$ & -9.01894700 & 3.78518600 & 0.40052200 \\
\hline C & -3.28969400 & -6.90693300 & 1.07600900 \\
\hline $\mathrm{H}$ & -3.89137200 & -7.12689100 & 1.95450900 \\
\hline $\mathrm{C}$ & -8.19516000 & -3.01639700 & 0.37422700 \\
\hline $\mathrm{H}$ & -9.10006200 & -3.61414600 & 0.43430200 \\
\hline C & 8.88690600 & -4.85141700 & 1.05816600 \\
\hline $\mathrm{H}$ & 9.50814700 & -5.04236500 & 0.17784700 \\
\hline $\mathrm{H}$ & 8.80516600 & -5.78335200 & 1.62528400 \\
\hline $\mathrm{H}$ & 9.42842000 & -4.13388900 & 1.68754400 \\
\hline C & -7.86723200 & -2.13198700 & 1.40333000 \\
\hline $\mathrm{H}$ & -8.51557900 & -2.04064900 & 2.27016700 \\
\hline C & -4.04066700 & -9.11185700 & 0.07751300 \\
\hline $\mathrm{H}$ & -4.24617500 & -9.50635400 & -0.92173500 \\
\hline $\mathrm{H}$ & -3.48257700 & -9.88211800 & 0.62451500 \\
\hline $\mathrm{H}$ & -4.99681000 & -8.97917900 & 0.59313900 \\
\hline $\mathrm{C}$ & -3.89337000 & 9.16146800 & -0.17663000 \\
\hline $\mathrm{H}$ & -3.84448200 & 9.66645800 & 0.79215900 \\
\hline $\mathrm{H}$ & -3.48467100 & 9.84976800 & -0.92685700 \\
\hline $\mathrm{H}$ & -4.94824000 & 9.00540900 & -0.42455000 \\
\hline $\mathrm{Se}$ & 4.81679000 & -0.04946800 & -0.17886500 \\
\hline
\end{tabular}


Figure S29: Cartesian coordinates of the S0 optimized structures of the compounds normalselenabenzosaphyrin6. \#Sum of imaginary frequencies=0; \#Thermal Free Energy (hartree) $=\mathbf{- 5 1 6 1 . 5 9 2 0 3 4}$.

\begin{tabular}{|c|c|c|c|}
\hline Atoms & $x$ & $Y$ & Z \\
\hline $\mathrm{C}$ & 3.96792100 & 1.32965500 & 0.07390600 \\
\hline $\mathrm{C}$ & 5.22497400 & 0.67694800 & 0.26440300 \\
\hline C & 5.22343600 & -0.68500800 & 0.27377800 \\
\hline C & 3.96494800 & -1.33737300 & 0.09145300 \\
\hline $\mathrm{C}$ & 0.55679900 & -4.11626500 & -0.17126100 \\
\hline $\mathrm{C}$ & 1.35325600 & -5.32448800 & -0.31793700 \\
\hline $\mathrm{C}$ & 2.64976000 & -4.93329500 & -0.26386900 \\
\hline $\mathrm{C}$ & 2.63792900 & -3.48343800 & -0.09147800 \\
\hline $\mathrm{N}$ & 1.37603100 & -3.00690900 & -0.05553700 \\
\hline C & 3.83996700 & -2.71872700 & 0.02774300 \\
\hline $\mathrm{C}$ & -2.04530500 & -0.69500700 & -0.03961100 \\
\hline $\mathrm{C}$ & -3.30083000 & -1.38244300 & 0.02708800 \\
\hline $\mathrm{C}$ & -3.00288800 & -2.75107900 & 0.00063200 \\
\hline $\mathrm{C}$ & -1.60419900 & -2.89420900 & -0.10157400 \\
\hline $\mathrm{N}$ & -1.04692000 & -1.62751400 & -0.12149200 \\
\hline $\mathrm{C}$ & -2.04186500 & 0.69713800 & -0.01685300 \\
\hline C & -3.29365300 & 1.38759500 & 0.07775700 \\
\hline C & -2.99479800 & 2.75520200 & 0.02641200 \\
\hline $\mathrm{C}$ & -1.59717400 & 2.89543600 & -0.09196500 \\
\hline $\mathrm{N}$ & -1.04240800 & 1.62760800 & -0.11020900 \\
\hline C & 0.56467600 & 4.11274100 & -0.20051900 \\
\hline C & 1.36250200 & 5.31698000 & -0.37081100 \\
\hline C & 2.65861600 & 4.92356100 & -0.32287000 \\
\hline C & 2.64508100 & 3.47623800 & -0.13059900 \\
\hline $\mathrm{N}$ & 1.38254900 & 3.00311400 & -0.07807000 \\
\hline $\mathrm{C}$ & 3.84605300 & 2.71036600 & -0.00753900 \\
\hline $\mathrm{C}$ & -0.84252000 & -4.09094700 & -0.16015400 \\
\hline $\mathrm{C}$ & -0.83456300 & 4.09050100 & -0.17312200 \\
\hline $\mathrm{C}$ & -4.55860500 & 0.69589800 & 0.16210200 \\
\hline $\mathrm{C}$ & -4.56247300 & -0.68730500 & 0.12968400 \\
\hline $\mathrm{C}$ & -1.59927500 & -5.37554900 & -0.20119900 \\
\hline $\mathrm{C}$ & -1.58891600 & 5.37621700 & -0.22135000 \\
\hline $\mathrm{C}$ & -5.80712800 & -1.50377600 & 0.23371000 \\
\hline C & -5.80219800 & 1.51763600 & 0.23055100 \\
\hline $\mathrm{C}$ & 5.11398800 & -3.51809800 & 0.09229000 \\
\hline C & 5.12233800 & 3.50705000 & 0.04172300 \\
\hline C & 5.47675300 & -4.19948500 & 1.26271600 \\
\hline $\mathrm{C}$ & 6.65238300 & -4.94658500 & 1.31912800 \\
\hline C & 7.50283100 & -5.04592800 & 0.20993100 \\
\hline $\mathrm{C}$ & 7.13825700 & -4.36317600 & -0.95711800 \\
\hline $\mathrm{C}$ & 5.96496800 & -3.61131000 & -1.01722500 \\
\hline $\mathrm{C}$ & 5.96942400 & 3.58302100 & -1.07299500 \\
\hline $\mathrm{C}$ & 7.14035400 & 4.33845200 & -1.03052700 \\
\hline C & 7.51018300 & 5.03855500 & 0.12545500 \\
\hline C & 6.66116700 & 4.96181200 & 1.23658600 \\
\hline $\mathrm{C}$ & 5.48659100 & 4.21073700 & 1.19760800 \\
\hline $\mathrm{C}$ & -2.49269600 & -5.65779800 & -1.24698000 \\
\hline $\mathrm{C}$ & -3.19508100 & -6.86044900 & -1.28270500 \\
\hline $\mathrm{C}$ & -3.04231600 & -7.82122500 & -0.27475100 \\
\hline
\end{tabular}

\begin{tabular}{|c|c|c|c|}
\hline Atoms & $x$ & Y & Z \\
\hline $\mathrm{C}$ & -2.15071900 & -7.53827800 & 0.76793700 \\
\hline C & -1.44113700 & -6.33994500 & 0.80683800 \\
\hline C & -1.41335500 & 6.35400600 & 0.77076800 \\
\hline C & -2.12548900 & 7.55090800 & 0.72964600 \\
\hline C & -3.03365800 & 7.82084800 & -0.30205300 \\
\hline C & -3.20964900 & 6.84333800 & -1.29008100 \\
\hline C & -2.50459800 & 5.64243900 & -1.25229500 \\
\hline C & -6.80215300 & 1.40148500 & -0.74792500 \\
\hline C & -7.95020800 & 2.19005800 & -0.69322000 \\
\hline C & -8.12203400 & 3.11262900 & 0.34027600 \\
\hline C & -7.13547500 & 3.24052100 & 1.31888800 \\
\hline C & -5.98534800 & 2.45287200 & 1.26260600 \\
\hline C & -6.14581800 & -2.42213600 & -0.77374600 \\
\hline C & -7.29923800 & -3.20045400 & -0.67460600 \\
\hline C & -8.13312700 & -3.08008300 & 0.43778400 \\
\hline C & -7.80502300 & -2.17491200 & 1.44864200 \\
\hline C & -6.65402600 & -1.39511900 & 1.34780700 \\
\hline $\mathrm{C}$ & 8.75836400 & -5.88382900 & 0.26503000 \\
\hline C & 8.79572400 & 5.82977400 & 0.17775900 \\
\hline C & -3.83289800 & -9.10742900 & -0.29699500 \\
\hline C & -3.77948900 & 9.13243100 & -0.36150300 \\
\hline $\mathrm{H}$ & -9.01700800 & 3.72663000 & 0.38238500 \\
\hline $\mathrm{H}$ & -7.26037600 & 3.95262400 & 2.12975300 \\
\hline $\mathrm{H}$ & -8.71078000 & 2.08549900 & -1.46180600 \\
\hline $\mathrm{H}$ & -6.67339600 & 0.68796700 & -1.55527800 \\
\hline $\mathrm{H}$ & -5.22333900 & 2.55295000 & 2.02992300 \\
\hline $\mathrm{H}$ & -8.44544800 & -2.07667300 & 2.32055000 \\
\hline $\mathrm{H}$ & -6.40310400 & -0.69411400 & 2.13720300 \\
\hline $\mathrm{H}$ & -9.03055200 & -3.68676600 & 0.51695900 \\
\hline $\mathrm{H}$ & -7.54708400 & -3.89904000 & -1.46887100 \\
\hline $\mathrm{H}$ & -5.50404700 & -2.51501700 & -1.64494300 \\
\hline $\mathrm{H}$ & -3.69730000 & 3.57136800 & 0.07099400 \\
\hline $\mathrm{H}$ & -3.70497100 & -3.56682700 & 0.05994200 \\
\hline $\mathrm{H}$ & -2.62064000 & -4.93437000 & -2.04629000 \\
\hline $\mathrm{H}$ & -3.86968500 & -7.05837300 & -2.11207800 \\
\hline $\mathrm{H}$ & -2.01156500 & -8.26489600 & 1.56470900 \\
\hline $\mathrm{H}$ & -0.76244200 & -6.13992800 & 1.63002700 \\
\hline $\mathrm{H}$ & -4.77944000 & -8.99953500 & 0.24747800 \\
\hline $\mathrm{H}$ & -3.28024700 & -9.92594600 & 0.17383400 \\
\hline $\mathrm{H}$ & 9.50200900 & -5.53939300 & -0.45949000 \\
\hline $\mathrm{H}$ & 9.21534300 & -5.85597100 & 1.25900800 \\
\hline $\mathrm{H}$ & 6.91481300 & -5.45893700 & 2.24165600 \\
\hline $\mathrm{H}$ & 4.83325100 & -4.13641200 & 2.13538100 \\
\hline $\mathrm{H}$ & 5.70304700 & -3.08975800 & -1.93323900 \\
\hline $\mathrm{H}$ & 7.78070900 & -4.42001300 & -1.83249200 \\
\hline $\mathrm{H}$ & 0.96622800 & -6.32243100 & -0.46024700 \\
\hline $\mathrm{H}$ & 3.53091900 & -5.55117000 & -0.34799200 \\
\hline $\mathrm{H}$ & 6.12650600 & -1.26395600 & 0.41365200 \\
\hline $\mathrm{H}$ & 6.12930600 & 1.25577500 & 0.39646800 \\
\hline
\end{tabular}




\begin{tabular}{cccc}
\hline Atoms & $\mathrm{X}$ & $\mathrm{Y}$ & $\mathrm{Z}$ \\
\hline $\mathrm{H}$ & 5.70089200 & 3.05149000 & -1.98132700 \\
$\mathrm{H}$ & 4.84165200 & 4.16935600 & 2.07048900 \\
$\mathrm{H}$ & 6.92134300 & 5.49814000 & 2.14592200 \\
$\mathrm{H}$ & 7.77544100 & 4.38791500 & -1.91184700 \\
$\mathrm{H}$ & 9.64606200 & 5.18853200 & 0.44186100 \\
$\mathrm{H}$ & 8.74505800 & 6.62676300 & 0.92526000 \\
$\mathrm{H}$ & 3.54033200 & 5.53825200 & -0.42305500 \\
$\mathrm{H}$ & 0.97658300 & 6.31386900 & -0.52309500 \\
$\mathrm{H}$ & -2.65541300 & 4.90409300 & -2.03363900 \\
$\mathrm{H}$ & -0.72458300 & 6.16257400 & 1.58763500 \\
$\mathrm{H}$ & -1.97740300 & 8.28517900 & 1.51772400 \\
\hline
\end{tabular}

\begin{tabular}{lrrr}
\hline Atoms & $\mathrm{X}$ & $\mathrm{Y}$ & $\mathrm{Z}$ \\
\hline $\mathrm{H}$ & -3.90791300 & 7.02442700 & -2.10346200 \\
$\mathrm{H}$ & -3.94527400 & 9.54442900 & 0.63834400 \\
$\mathrm{H}$ & -0.03461400 & -1.51048400 & -0.11740500 \\
$\mathrm{H}$ & -0.03046200 & 1.50840500 & -0.12171800 \\
$\mathrm{H}$ & 8.54396600 & -6.93547900 & 0.03693600 \\
$\mathrm{H}$ & 9.02449200 & 6.28660400 & -0.79001500 \\
H & -4.07939700 & -9.40836900 & -1.31949700 \\
H & -3.21754200 & 9.88208600 & -0.93275000 \\
H & -4.75312100 & 9.01794700 & -0.84714000 \\
Se & 2.60025100 & -0.00325200 & -0.05342600 \\
\hline
\end{tabular}

Figure S30: Cartesian coordinates of the S0 optimized structures of the compounds invertedtellurabenzosaphyrin. \#Sum of imaginary frequencies $=0$; \#Thermal free Energy (hartree)

-2691.582070.

\begin{tabular}{lrrr}
\hline Atoms & $\mathrm{X}$ & $\mathrm{Y}$ & $\mathrm{Z}$ \\
\hline $\mathrm{N}$ & -0.98318300 & 1.65805100 & -0.06682900 \\
$\mathrm{H}$ & 0.03242900 & 1.56589300 & -0.06358400 \\
$\mathrm{~N}$ & 1.38182600 & 3.11182400 & -0.17627300 \\
$\mathrm{~N}$ & -1.00008200 & -1.66008700 & -0.03191400 \\
$\mathrm{H}$ & 0.01613100 & -1.57778400 & -0.04893800 \\
$\mathrm{~N}$ & 1.34264300 & -3.13238500 & -0.25961500 \\
$\mathrm{C}$ & -1.61841100 & 5.41649100 & -0.14002100 \\
$\mathrm{C}$ & 0.56595500 & 4.22278600 & -0.20827000 \\
$\mathrm{C}$ & 2.64392600 & 3.56763100 & -0.26980500 \\
$\mathrm{C}$ & 5.11907300 & 3.35793500 & 0.08942700 \\
$\mathrm{C}$ & 3.75178300 & 1.37384300 & -0.56373300 \\
$\mathrm{C}$ & 2.63278500 & -0.69202000 & -1.14291900 \\
$\mathrm{H}$ & 1.80930300 & -1.25364200 & -1.56671600 \\
$\mathrm{C}$ & 1.36759800 & 5.43080900 & -0.34275000 \\
$\mathrm{H}$ & 0.98795300 & 6.43899800 & -0.42506000 \\
$\mathrm{C}$ & 7.58079400 & 4.52874200 & 0.80257000 \\
$\mathrm{C}$ & -0.82826800 & 4.15142800 & -0.13527500 \\
$\mathrm{C}$ & 5.25122100 & 4.08589500 & 1.28536800 \\
$\mathrm{H}$ & 4.39227100 & 4.18448000 & 1.94185200 \\
$\mathrm{C}$ & 2.64244900 & 0.67642900 & -1.13395500 \\
$\mathrm{H}$ & 1.82676000 & 1.25332400 & -1.55259600 \\
$\mathrm{C}$ & -1.55485100 & 2.92484200 & -0.05753500 \\
$\mathrm{C}$ & -1.97294300 & 0.70592700 & 0.01424000 \\
$\mathrm{C}$ & 5.08329400 & -3.39440000 & 0.08484500 \\
$\mathrm{C}$ & 6.24382200 & 3.23688500 & -0.74614600 \\
$\mathrm{H}$ & 6.15016800 & 2.70308900 & -1.68701500 \\
$\mathrm{C}$ & 6.47060600 & 4.66214200 & 1.63930500 \\
$\mathrm{H}$ & 6.55485500 & 5.21213500 & 2.57236800 \\
$\mathrm{C}$ & -3.23219900 & 1.39125800 & 0.08386900 \\
$\mathrm{C}$ & 1.32094500 & -5.45562800 & -0.19367100 \\
$\mathrm{H}$ & 0.94011100 & -6.46529100 & -0.14204700 \\
$\mathrm{C}$ & 0.52214600 & -4.23835000 & -0.20437300 \\
$\mathrm{C}$ & -1.44560400 & 6.37578600 & 0.87076300 \\
$\mathrm{H}$ & -0.74141100 & 6.17942000 & 1.67346500 \\
\hline & & & \\
\hline
\end{tabular}

\begin{tabular}{|c|c|c|c|}
\hline Atoms & $x$ & $Y$ & Z \\
\hline $\mathrm{C}$ & 2.66565200 & 5.02402200 & -0.36085700 \\
\hline $\mathrm{H}$ & 3.55071800 & 5.63538800 & -0.46640900 \\
\hline C & -2.55151200 & 5.68901100 & -1.15388000 \\
\hline $\mathrm{H}$ & -2.69832400 & 4.96644500 & -1.95077700 \\
\hline C & 6.19547300 & -3.32090000 & -0.77228800 \\
\hline $\mathrm{H}$ & 6.09151000 & -2.82610800 & -1.73327100 \\
\hline C & -1.98136300 & -0.69701500 & 0.00355700 \\
\hline C & 3.81941500 & 2.72883600 & -0.28346000 \\
\hline $\mathrm{C}$ & 5.22850400 & -4.06631700 & 1.31141400 \\
\hline $\mathrm{H}$ & 4.37951100 & -4.12693700 & 1.98529000 \\
\hline C & 7.46254700 & 3.81680200 & -0.39259600 \\
\hline $\mathrm{H}$ & 8.31694200 & 3.71966700 & -1.05646700 \\
\hline C & -2.94803000 & 2.76325500 & 0.04272200 \\
\hline $\mathrm{H}$ & -3.66501400 & 3.56715900 & 0.07550800 \\
\hline C & 3.78491200 & -2.76584800 & -0.30001900 \\
\hline C & -5.76290600 & -1.48357200 & 0.13827700 \\
\hline C & 3.73201300 & -1.40995600 & -0.57946300 \\
\hline C & -2.18020500 & 7.56031400 & 0.86592700 \\
\hline $\mathrm{H}$ & -2.03417300 & 8.28012700 & 1.66782800 \\
\hline C & 2.60535200 & -3.59730300 & -0.27965000 \\
\hline C & 2.62057800 & -5.05641300 & -0.23753900 \\
\hline $\mathrm{H}$ & 3.50373300 & -5.67908200 & -0.25170800 \\
\hline C & -1.67012900 & -5.41180500 & -0.05430600 \\
\hline C & -1.58479700 & -2.92041600 & -0.03406100 \\
\hline C & -0.86956400 & -4.15450600 & -0.09862700 \\
\hline C & 7.41486700 & -3.89575200 & -0.41195900 \\
\hline $\mathrm{H}$ & 8.25959800 & -3.83594400 & -1.09241400 \\
\hline C & -5.74323700 & 1.53658500 & 0.17379500 \\
\hline C & -2.97943700 & -2.74361900 & 0.00074100 \\
\hline $\mathrm{H}$ & -3.70527500 & -3.54028500 & -0.00238100 \\
\hline C & -4.50198300 & 0.70941200 & 0.13129500 \\
\hline C & -3.27906200 & 6.87742700 & -1.15513700 \\
\hline $\mathrm{H}$ & -3.99117200 & 7.06395400 & -1.95535600 \\
\hline C & -7.86133100 & 2.23012100 & -0.80326500 \\
\hline
\end{tabular}




\begin{tabular}{lrrr}
\hline Atoms & $\mathrm{X}$ & $\mathrm{Y}$ & $\mathrm{Z}$ \\
\hline $\mathrm{H}$ & -8.59916900 & 2.13966300 & -1.59566400 \\
$\mathrm{C}$ & -4.51146000 & -0.67221300 & 0.08597400 \\
$\mathrm{C}$ & -3.10763100 & 7.83587200 & -0.14736700 \\
$\mathrm{C}$ & -3.25016500 & -1.36846400 & 0.02486300 \\
$\mathrm{C}$ & -6.71401100 & 1.43851700 & -0.83595700 \\
$\mathrm{H}$ & -6.56357500 & 0.73634500 & -1.64972700 \\
$\mathrm{C}$ & -5.95460700 & 2.45642500 & 1.21462900 \\
$\mathrm{H}$ & -5.21523400 & 2.54244600 & 2.00570600 \\
$\mathrm{C}$ & 7.54585000 & -4.55395200 & 0.81217600 \\
$\mathrm{C}$ & -1.62920600 & -6.32723400 & -1.11793800 \\
$\mathrm{H}$ & -1.02154000 & -6.10168400 & -1.98895400 \\
$\mathrm{C}$ & 6.44846300 & -4.63753400 & 1.67199100 \\
$\mathrm{H}$ & 6.54308600 & -5.14449700 & 2.62814800 \\
$\mathrm{C}$ & -2.37303400 & -7.50570400 & -1.07645600 \\
$\mathrm{H}$ & -2.33115900 & -8.19155700 & -1.91921900 \\
$\mathrm{C}$ & -6.07456200 & -2.38472600 & -0.89365600 \\
$\mathrm{H}$ & -5.40533900 & -2.46978200 & -1.74498400 \\
$\mathrm{C}$ & -2.48254600 & -5.72041500 & 1.04956500 \\
$\mathrm{H}$ & -2.52554600 & -5.03162400 & 1.88769100 \\
$\mathrm{C}$ & -6.64522200 & -1.38496400 & 1.22589000 \\
$\mathrm{H}$ & -6.41654000 & -0.69610500 & 2.03285900 \\
$\mathrm{C}$ & -3.17887400 & -7.81752900 & 0.02577400 \\
$\mathrm{C}$ & -7.10381800 & 3.24736400 & 1.24871300 \\
\hline & & &
\end{tabular}

\begin{tabular}{crrr}
\hline Atoms & $\mathrm{X}$ & $\mathrm{Y}$ & $\mathrm{Z}$ \\
\hline $\mathrm{H}$ & -7.25082500 & 3.94757600 & 2.06641400 \\
$\mathrm{C}$ & -7.23539000 & -3.15743900 & -0.84309300 \\
$\mathrm{H}$ & -7.46097400 & -3.84328700 & -1.65505100 \\
$\mathrm{C}$ & -8.06138800 & 3.13754300 & 0.23902400 \\
$\mathrm{H}$ & -8.95570500 & 3.75373000 & 0.26392400 \\
$\mathrm{C}$ & -3.22026600 & -6.90147400 & 1.08602000 \\
$\mathrm{H}$ & -3.83747100 & -7.11640800 & 1.95509500 \\
$\mathrm{C}$ & -8.10460600 & -3.04736200 & 0.24357300 \\
$\mathrm{H}$ & -9.00812300 & -3.64909100 & 0.28456900 \\
$\mathrm{C}$ & -7.80423400 & -2.15846500 & 1.27774500 \\
$\mathrm{H}$ & -8.47280200 & -2.06784200 & 2.12936800 \\
$\mathrm{C}$ & -3.96018100 & -9.10875500 & 0.08320300 \\
H & -4.15799200 & -9.50185400 & -0.91830000 \\
H & -3.40893900 & -9.88121200 & 0.63426100 \\
H & -4.92047600 & -8.97381800 & 0.59064600 \\
$\mathrm{C}$ & -3.87825500 & 9.13459300 & -0.16707700 \\
$\mathrm{H}$ & -4.05640300 & 9.51007100 & 0.84516300 \\
$\mathrm{H}$ & -3.32798100 & 9.91353000 & -0.71002500 \\
H & -4.84739100 & 9.01785400 & -0.66124000 \\
Te & 5.26656700 & -0.03167500 & -0.05716100 \\
H & 8.49522000 & -5.00063900 & 1.09330900 \\
H & 8.52994700 & 4.97920600 & 1.07834300 \\
\hline & & &
\end{tabular}

Figure S31: Cartesian coordinates of the S0 optimized structures of the compounds normaltellurabenzosaphyrin7. \#Sum of imaginary frequencies $=0$; \#Thermal free Energy (hartree)

$-2691.595374$.

\begin{tabular}{crrr}
\hline Atoms & $\mathrm{X}$ & $\mathrm{Y}$ & $\mathrm{Z}$ \\
\hline $\mathrm{C}$ & 4.17908700 & 1.38475500 & 0.06811700 \\
$\mathrm{C}$ & 5.41342000 & 0.67007000 & 0.18458800 \\
$\mathrm{C}$ & 5.40957000 & -0.69773900 & 0.19445400 \\
$\mathrm{C}$ & 4.17125000 & -1.40704000 & 0.08774500 \\
$\mathrm{C}$ & 0.76286600 & -4.13806400 & -0.10093100 \\
$\mathrm{C}$ & 1.53899000 & -5.36468200 & -0.20282200 \\
$\mathrm{C}$ & 2.84375100 & -4.99759000 & -0.15518200 \\
$\mathrm{C}$ & 2.86072000 & -3.54515600 & -0.03056500 \\
$\mathrm{~N}$ & 1.60762300 & -3.04209300 & -0.01282900 \\
$\mathrm{C}$ & 4.07582800 & -2.78807000 & 0.04899400 \\
$\mathrm{C}$ & -1.84000500 & -0.69344100 & -0.07696400 \\
$\mathrm{C}$ & -3.09688700 & -1.37932400 & -0.01770700 \\
$\mathrm{C}$ & -2.80152400 & -2.75036600 & -0.01896000 \\
$\mathrm{C}$ & -1.40188200 & -2.89863900 & -0.09719500 \\
$\mathrm{~N}$ & -0.84471000 & -1.63272400 & -0.13324600 \\
$\mathrm{C}$ & -1.83484900 & 0.70076200 & -0.05587600 \\
$\mathrm{C}$ & -3.08615100 & 1.39350800 & 0.03182200 \\
$\mathrm{C}$ & -2.78480900 & 2.76285100 & 0.00464200 \\
$\mathrm{C}$ & -1.38561000 & 2.90372500 & -0.09170700 \\
$\mathrm{~N}$ & -0.83532200 & 1.63476200 & -0.12515400 \\
$\mathrm{C}$ & 0.78485600 & 4.13188500 & -0.13758100 \\
$\mathrm{C}$ & 1.56690300 & 5.35228100 & -0.26562100 \\
$\mathrm{C}$ & 2.87001500 & 4.97857400 & -0.22341900 \\
$\mathrm{C}$ & 2.88001700 & 3.52824800 & -0.07608600 \\
\hline
\end{tabular}

\begin{tabular}{crrr}
\hline Atoms & $\mathrm{X}$ & $\mathrm{Y}$ & $\mathrm{Z}$ \\
\hline $\mathrm{N}$ & 1.62437100 & 3.03258900 & -0.04113300 \\
$\mathrm{C}$ & 4.09138100 & 2.76560400 & 0.00883700 \\
$\mathrm{C}$ & -0.63703900 & -4.09665200 & -0.11218200 \\
$\mathrm{C}$ & -0.61543300 & 4.09801900 & -0.13162900 \\
$\mathrm{C}$ & -4.35268600 & 0.70265600 & 0.09690200 \\
$\mathrm{C}$ & -4.35835100 & -0.68118500 & 0.06454200 \\
$\mathrm{C}$ & -1.40235800 & -5.37793800 & -0.13004700 \\
$\mathrm{C}$ & -1.37396300 & 5.38307900 & -0.15788900 \\
$\mathrm{C}$ & -5.60574900 & -1.49546800 & 0.15029200 \\
$\mathrm{C}$ & -5.59653300 & 1.52530700 & 0.14929000 \\
$\mathrm{C}$ & 5.35259900 & -3.58361100 & 0.08890800 \\
$\mathrm{C}$ & 5.37249300 & 3.55461500 & 0.03246000 \\
$\mathrm{C}$ & 5.74679300 & -4.24226100 & 1.26331300 \\
$\mathrm{C}$ & 6.92888900 & -4.98355800 & 1.30136100 \\
$\mathrm{C}$ & 7.73408900 & -5.07941400 & 0.16458800 \\
$\mathrm{C}$ & 7.35115900 & -4.42845000 & -1.00966000 \\
C & 6.17004800 & -3.68519300 & -1.04668600 \\
C & 6.19109400 & 3.62759800 & -1.10451900 \\
C & 7.37587400 & 4.36561000 & -1.08269700 \\
C & 7.76153300 & 5.03955300 & 0.07759100 \\
C & 6.95538000 & 4.97203600 & 1.21576200 \\
C & 5.76956900 & 4.23620100 & 1.19281200 \\
C & -2.26448500 & -5.69131400 & -1.19313800 \\
C & -2.97560000 & -6.88977200 & -1.20756600 \\
\hline
\end{tabular}




\begin{tabular}{lrrr}
\hline Atoms & $\mathrm{X}$ & $\mathrm{Y}$ & $\mathrm{Z}$ \\
\hline $\mathrm{C}$ & -2.86257500 & -7.81413200 & -0.16060700 \\
$\mathrm{C}$ & -2.00226600 & -7.49951900 & 0.89963800 \\
$\mathrm{C}$ & -1.28403700 & -6.30538300 & 0.91757000 \\
$\mathrm{C}$ & -1.23104700 & 6.32763300 & 0.87121600 \\
$\mathrm{C}$ & -1.94815700 & 7.52257600 & 0.84974700 \\
$\mathrm{C}$ & -2.82852800 & 7.82285600 & -0.19796500 \\
$\mathrm{C}$ & -2.97221600 & 6.87784200 & -1.22269400 \\
$\mathrm{C}$ & -2.26206900 & 5.67904400 & -1.20490600 \\
$\mathrm{C}$ & -6.58057300 & 1.41409700 & -0.84615300 \\
$\mathrm{C}$ & -7.72909200 & 2.20355600 & -0.80656000 \\
$\mathrm{C}$ & -7.91712700 & 3.12180000 & 0.22849600 \\
$\mathrm{C}$ & -6.94641400 & 3.24447300 & 1.22402300 \\
$\mathrm{C}$ & -5.79581400 & 2.45587700 & 1.18295000 \\
$\mathrm{C}$ & -5.92608500 & -2.41872900 & -0.85919200 \\
$\mathrm{C}$ & -7.08218400 & -3.19578700 & -0.77818500 \\
$\mathrm{C}$ & -7.93708400 & -3.06917600 & 0.31793100 \\
$\mathrm{C}$ & -7.62723700 & -2.15914400 & 1.33073500 \\
$\mathrm{C}$ & -6.47355700 & -1.38054000 & 1.24797000 \\
$\mathrm{C}$ & -3.66266300 & -9.09498200 & -0.16139800 \\
$\mathrm{C}$ & -3.57924000 & 9.13278300 & -0.23496900 \\
H & -8.81238200 & 3.73635500 & 0.25877500 \\
H & -7.08418300 & 3.95292900 & 2.03620000 \\
H & -8.47726800 & 2.10305900 & -1.58796300 \\
H & -6.43925400 & 0.70371800 & -1.65443900 \\
H & -5.04648200 & 2.55162000 & 1.96344200 \\
H & -8.28401100 & -2.05626600 & 2.19004600 \\
H & -6.23714300 & -0.67585100 & 2.03876600 \\
H & -8.83658500 & -3.67473300 & 0.38306100 \\
H & -7.31566600 & -3.89808600 & -1.57373200 \\
H & -5.26771500 & -2.51668300 & -1.71757000 \\
H & -3.48754500 & 3.57865400 & 0.05473200 \\
H & -3.50732500 & -3.56274300 & 0.04504800 \\
& -2.36125100 & -4.99636000 & -2.02183000 \\
\hline & & & \\
\hline
\end{tabular}

\begin{tabular}{crrr}
\hline Atoms & $\mathrm{X}$ & $\mathrm{Y}$ & $\mathrm{Z}$ \\
\hline $\mathrm{H}$ & -3.62537600 & -7.11330900 & -2.05031200 \\
$\mathrm{H}$ & -1.89448200 & -8.19764300 & 1.72635800 \\
$\mathrm{H}$ & -0.62925900 & -6.08035900 & 1.75384000 \\
$\mathrm{H}$ & -4.62326400 & -8.96157000 & 0.35211900 \\
$\mathrm{H}$ & -3.13012300 & -9.90089900 & 0.35266500 \\
$\mathrm{H}$ & 7.22103900 & -5.48454800 & 2.22008300 \\
$\mathrm{H}$ & 5.12177000 & -4.16793500 & 2.14852200 \\
$\mathrm{H}$ & 5.87195500 & -3.18084300 & -1.96130300 \\
$\mathrm{H}$ & 7.97065300 & -4.49994500 & -1.89934600 \\
$\mathrm{H}$ & 1.13365000 & -6.35926900 & -0.31467400 \\
$\mathrm{H}$ & 3.71330900 & -5.63516900 & -0.21352700 \\
$\mathrm{H}$ & 6.33690900 & -1.25293900 & 0.28176800 \\
$\mathrm{H}$ & 6.34386300 & 1.22121700 & 0.26415800 \\
$\mathrm{H}$ & 5.89079700 & 3.10529400 & -2.00825800 \\
$\mathrm{H}$ & 5.14366400 & 4.18400000 & 2.07897300 \\
$\mathrm{H}$ & 7.24973700 & 5.49101800 & 2.12373000 \\
$\mathrm{H}$ & 7.99607200 & 4.41506600 & -1.97338900 \\
$\mathrm{H}$ & 3.74251500 & 5.61026300 & -0.29920500 \\
$\mathrm{H}$ & 1.16629700 & 6.34734800 & -0.38943600 \\
$\mathrm{H}$ & -2.38759400 & 4.96607300 & -2.01408500 \\
$\mathrm{H}$ & -0.56359500 & 6.11227500 & 1.70000500 \\
$\mathrm{H}$ & -1.82589900 & 8.23114000 & 1.66547300 \\
$\mathrm{H}$ & -3.64872700 & 7.08298400 & -2.04883500 \\
$\mathrm{H}$ & -3.77014900 & 9.51361000 & 0.77284600 \\
$\mathrm{H}$ & 0.16482000 & -1.51935700 & -0.12984300 \\
$\mathrm{H}$ & 0.17337500 & 1.51595200 & -0.14268400 \\
$\mathrm{H}$ & -3.88285500 & -9.42911400 & -1.17971500 \\
$\mathrm{H}$ & -3.00772100 & 9.90175400 & -0.77011800 \\
$\mathrm{H}$ & -4.54085800 & 9.02923800 & -0.74662100 \\
Te & 2.58976100 & -0.00724100 & 0.00761600 \\
$\mathrm{H}$ & 8.65343400 & -5.65730800 & 0.19370400 \\
$\mathrm{H}$ & 8.68381000 & 5.61323900 & 0.09488700 \\
\hline & & & \\
& & & \\
& & &
\end{tabular}

Figure S32: Cartesian coordinates of the S0 optimized structures of the compounds inverted thiabenzosaphyrin5'. \#Sum of imaginary frequencies=0; \#Thermal Free Energy (hartree) = 3160.308314 .

\begin{tabular}{crcc}
\hline Atoms & $\mathrm{X}$ & $\mathrm{Y}$ & $\mathrm{Z}$ \\
\hline $\mathrm{N}$ & -0.88531400 & 1.65517200 & -0.10936600 \\
$\mathrm{H}$ & 0.13036500 & 1.55648000 & -0.13009600 \\
$\mathrm{~N}$ & 1.47834300 & 3.05463900 & -0.25657700 \\
$\mathrm{~N}$ & -0.91903900 & -1.65434200 & -0.07691800 \\
$\mathrm{H}$ & 0.09764500 & -1.57674000 & -0.12167100 \\
$\mathrm{~N}$ & 1.40334900 & -3.09521400 & -0.35031600 \\
$\mathrm{C}$ & -1.46579600 & 5.42190400 & -0.13652300 \\
$\mathrm{C}$ & 0.69573300 & 4.18411500 & -0.26140600 \\
$\mathrm{C}$ & 2.75537600 & 3.47211900 & -0.37463200 \\
$\mathrm{C}$ & 5.22451900 & 3.09527800 & -0.05841700 \\
\hline
\end{tabular}

\begin{tabular}{crrr}
\hline Atoms & $\mathrm{X}$ & $\mathrm{Y}$ & $\mathrm{Z}$ \\
\hline $\mathrm{C}$ & 3.68616100 & 1.23702300 & -0.74833300 \\
$\mathrm{C}$ & 2.54269700 & -0.70406100 & -1.41701600 \\
H & 1.77176600 & -1.30488100 & -1.87721100 \\
$\mathrm{C}$ & 1.53043900 & 5.37015700 & -0.40119200 \\
H & 1.17921200 & 6.38968900 & -0.46870700 \\
$\mathrm{C}$ & 7.79813800 & 4.08777600 & 0.63295700 \\
$\mathrm{C}$ & -0.70151100 & 4.14242400 & -0.16015600 \\
$\mathrm{C}$ & 5.41123400 & 3.90547500 & 1.07560200 \\
H & 4.55839400 & 4.14358600 & 1.70308900 \\
$\mathrm{C}$ & 2.55853700 & 0.66386000 & -1.40543600 \\
\hline
\end{tabular}




\begin{tabular}{|c|c|c|c|}
\hline Atoms & $x$ & $Y$ & Z \\
\hline $\mathrm{H}$ & 1.80226200 & 1.28856000 & -1.85843600 \\
\hline $\mathrm{C}$ & -1.44373300 & 2.92680300 & -0.07986100 \\
\hline C & -1.87998800 & 0.71128600 & -0.00999300 \\
\hline C & 5.15120000 & -3.18747900 & -0.04947100 \\
\hline C & 6.35456300 & 2.79731800 & -0.84166300 \\
\hline $\mathrm{H}$ & 6.23670000 & 2.19576300 & -1.73738300 \\
\hline $\mathrm{C}$ & 6.67319200 & 4.38964000 & 1.41104000 \\
\hline $\mathrm{H}$ & 6.78733300 & 5.00889200 & 2.29740900 \\
\hline C & -3.13226800 & 1.40632800 & 0.08979900 \\
\hline C & 1.43136700 & -5.41349900 & -0.27081500 \\
\hline $\mathrm{H}$ & 1.07246800 & -6.43055300 & -0.20830300 \\
\hline C & 0.60729000 & -4.21191100 & -0.27319700 \\
\hline $\mathrm{C}$ & -1.23794100 & 6.37781900 & 0.86508400 \\
\hline $\mathrm{H}$ & -0.51032200 & 6.16762200 & 1.64270100 \\
\hline $\mathrm{C}$ & 2.81640100 & 4.92746200 & -0.45002800 \\
\hline $\mathrm{H}$ & 3.71533300 & 5.51472600 & -0.57354900 \\
\hline $\mathrm{C}$ & -2.42900700 & 5.71420300 & -1.11751400 \\
\hline $\mathrm{H}$ & -2.61662200 & 4.99545300 & -1.90902400 \\
\hline $\mathrm{C}$ & 6.28445800 & -2.95228000 & -0.84855300 \\
\hline $\mathrm{H}$ & 6.17545800 & -2.39399200 & -1.77314700 \\
\hline $\mathrm{C}$ & -1.89604800 & -0.68935000 & -0.02119400 \\
\hline C & 3.88170800 & 2.58209000 & -0.42596700 \\
\hline C & 5.32452400 & -3.93830100 & 1.12618800 \\
\hline $\mathrm{H}$ & 4.46869600 & -4.12562300 & 1.76670100 \\
\hline C & 7.61209500 & 3.28634700 & -0.50184200 \\
\hline $\mathrm{H}$ & 8.46424100 & 3.04999600 & -1.13438700 \\
\hline $\mathrm{C}$ & -2.83820000 & 2.77504600 & 0.04934600 \\
\hline $\mathrm{H}$ & -3.54749600 & 3.58462800 & 0.10306000 \\
\hline $\mathrm{C}$ & 3.81863500 & -2.66113700 & -0.44294300 \\
\hline $\mathrm{C}$ & -5.67650000 & -1.45700100 & 0.20102900 \\
\hline $\mathrm{C}$ & 3.65529700 & -1.31222800 & -0.76609200 \\
\hline $\mathrm{C}$ & -1.94730000 & 7.57813500 & 0.88409000 \\
\hline $\mathrm{H}$ & -1.75741800 & 8.29577000 & 1.67833300 \\
\hline $\mathrm{C}$ & 2.67892800 & -3.53264100 & -0.39284500 \\
\hline $\mathrm{C}$ & 2.72268500 & -4.99004300 & -0.34094300 \\
\hline $\mathrm{H}$ & 3.61679100 & -5.59620800 & -0.37270300 \\
\hline $\mathrm{C}$ & -1.57276100 & -5.40780100 & -0.06803400 \\
\hline $\mathrm{C}$ & -1.50427200 & -2.91354400 & -0.06179900 \\
\hline $\mathrm{C}$ & -0.78617900 & -4.14403700 & -0.13687300 \\
\hline $\mathrm{C}$ & 7.53333700 & -3.44762300 & -0.48551300 \\
\hline $\mathrm{H}$ & 8.38858900 & -3.26122900 & -1.13047700 \\
\hline $\mathrm{C}$ & -5.64071300 & 1.56204500 & 0.23240400 \\
\hline $\mathrm{C}$ & -2.89978500 & -2.73227300 & 0.00395700 \\
\hline $\mathrm{H}$ & -3.62725700 & -3.52729900 & 0.01965300 \\
\hline $\mathrm{C}$ & -4.40448800 & 0.72889400 & 0.16409600 \\
\hline $\mathrm{C}$ & -3.13094900 & 6.91610700 & -1.09463700 \\
\hline $\mathrm{H}$ & -3.86627900 & 7.11714800 & -1.86998500 \\
\hline $\mathrm{C}$ & -7.77326500 & 2.26714800 & -0.70273400 \\
\hline $\mathrm{H}$ & -8.52658800 & 2.18124400 & -1.48071600 \\
\hline $\mathrm{C}$ & -4.42216800 & -0.65238800 & 0.11954900 \\
\hline
\end{tabular}

\begin{tabular}{|c|c|c|c|}
\hline Atoms & $x$ & $Y$ & Z \\
\hline C & -2.90396000 & 7.87191900 & -0.09439700 \\
\hline $\mathrm{C}$ & -3.16611400 & -1.35745500 & 0.03059500 \\
\hline $\mathrm{C}$ & -6.63105400 & 1.47012300 & -0.75828900 \\
\hline $\mathrm{H}$ & -6.49971700 & 0.76831100 & -1.57543000 \\
\hline $\mathrm{C}$ & -5.82717600 & 2.48174500 & 1.27765200 \\
\hline $\mathrm{H}$ & -5.07233900 & 2.56317800 & 2.05423100 \\
\hline $\mathrm{C}$ & 7.70613300 & -4.19154300 & 0.68967900 \\
\hline $\mathrm{C}$ & -1.52886600 & -6.33948200 & -1.11740500 \\
\hline $\mathrm{H}$ & -0.93015200 & -6.12052800 & -1.99607100 \\
\hline $\mathrm{C}$ & 6.57778900 & -4.42885900 & 1.48487600 \\
\hline $\mathrm{H}$ & 6.68243300 & -5.00201700 & 2.40283300 \\
\hline $\mathrm{C}$ & -2.25907000 & -7.52485800 & -1.05339900 \\
\hline $\mathrm{H}$ & -2.21488300 & -8.22276800 & -1.88592800 \\
\hline C & 9.17024800 & 4.58645700 & 1.01699000 \\
\hline $\mathrm{H}$ & 9.11389600 & 5.52311300 & 1.57939800 \\
\hline $\mathrm{H}$ & 9.79578500 & 4.75618700 & 0.13534300 \\
\hline $\mathrm{H}$ & 9.69289700 & 3.85785900 & 1.64961400 \\
\hline $\mathrm{C}$ & -6.01626100 & -2.35664800 & -0.82286100 \\
\hline $\mathrm{H}$ & -5.36727500 & -2.44524400 & -1.68913300 \\
\hline $\mathrm{C}$ & -2.37502300 & -5.70922100 & 1.04511200 \\
\hline $\mathrm{H}$ & -2.41918600 & -5.00934500 & 1.87370000 \\
\hline $\mathrm{C}$ & -6.53260200 & -1.35425700 & 1.30857400 \\
\hline $\mathrm{H}$ & -6.28170800 & -0.66673500 & 2.10982100 \\
\hline $\mathrm{C}$ & -3.05507100 & -7.82849100 & 0.05786500 \\
\hline $\mathrm{C}$ & -6.97135600 & 3.27802500 & 1.33468200 \\
\hline $\mathrm{H}$ & -7.09901600 & 3.97796700 & 2.15564500 \\
\hline $\mathrm{C}$ & -7.17920200 & -3.12321900 & -0.74537100 \\
\hline $\mathrm{H}$ & -7.42683400 & -3.80797300 & -1.55159700 \\
\hline $\mathrm{C}$ & -7.94852800 & 3.17413300 & 0.34385400 \\
\hline $\mathrm{H}$ & -8.83895900 & 3.79466700 & 0.38670400 \\
\hline $\mathrm{C}$ & -3.09987500 & -6.89691100 & 1.10381900 \\
\hline $\mathrm{H}$ & -3.70928100 & -7.10512900 & 1.97984000 \\
\hline $\mathrm{C}$ & -8.02222700 & -3.00888800 & 0.36081700 \\
\hline $\mathrm{H}$ & -8.92734300 & -3.60610900 & 0.42280500 \\
\hline $\mathrm{C}$ & 9.06903300 & -4.69719600 & 1.09724400 \\
\hline $\mathrm{H}$ & 9.68307600 & -4.94106800 & 0.22492800 \\
\hline $\mathrm{H}$ & 8.99428600 & -5.59247800 & 1.72147300 \\
\hline $\mathrm{H}$ & 9.61443900 & -3.94119200 & 1.67618300 \\
\hline $\mathrm{C}$ & -7.69357100 & -2.12186400 & 1.38744400 \\
\hline $\mathrm{H}$ & -8.34153900 & -2.02798900 & 2.25428900 \\
\hline $\mathrm{C}$ & -3.82175500 & -9.12681700 & 0.13955600 \\
\hline $\mathrm{H}$ & -4.02446400 & -9.53508700 & -0.85475200 \\
\hline $\mathrm{H}$ & -3.25743400 & -9.88611400 & 0.69545700 \\
\hline $\mathrm{H}$ & -4.77868800 & -8.99529000 & 0.65390600 \\
\hline $\mathrm{C}$ & -3.65496800 & 9.18188200 & -0.08932300 \\
\hline $\mathrm{H}$ & -3.57384500 & 9.68595600 & 0.87776100 \\
\hline $\mathrm{H}$ & -3.26294900 & 9.86694400 & -0.85123200 \\
\hline $\mathrm{H}$ & -4.71803200 & 9.03477300 & -0.30624900 \\
\hline $\mathrm{S}$ & 4.79995000 & -0.05408800 & -0.26249100 \\
\hline
\end{tabular}


Figure S33: Cartesian coordinates of the S0 optimized structures of the compounds normal thiabenzosaphyrin5. \#Sum of imaginary frequencies=0; \#Thermal Free Energy (hartree) =3160.389176.

\begin{tabular}{crrr}
\hline Atoms & $\mathrm{X}$ & $\mathrm{Y}$ & $\mathrm{Z}$ \\
\hline $\mathrm{C}$ & 4.08592100 & 1.28084900 & 0.10030200 \\
$\mathrm{C}$ & 5.35465800 & 0.67407000 & 0.36009200 \\
$\mathrm{C}$ & 5.35237300 & -0.68639000 & 0.37006700 \\
$\mathrm{C}$ & 4.08174000 & -1.29261400 & 0.11802800 \\
$\mathrm{C}$ & 0.64608800 & -4.09230400 & -0.22624100 \\
$\mathrm{C}$ & 1.45569300 & -5.28549300 & -0.41455700 \\
$\mathrm{C}$ & 2.74720000 & -4.87998400 & -0.35440700 \\
$\mathrm{C}$ & 2.71988700 & -3.43477500 & -0.13512700 \\
$\mathrm{~N}$ & 1.45146800 & -2.97690400 & -0.08289500 \\
$\mathrm{C}$ & 3.91979500 & -2.67362500 & 0.02275300 \\
$\mathrm{C}$ & -1.96084500 & -0.69411300 & -0.02294000 \\
$\mathrm{C}$ & -3.21616200 & -1.38176300 & 0.05269400 \\
$\mathrm{C}$ & -2.91903100 & -2.75005600 & 0.01000200 \\
$\mathrm{C}$ & -1.52096000 & -2.89097200 & -0.11102900 \\
$\mathrm{~N}$ & -0.96383200 & -1.62491300 & -0.12354900 \\
$\mathrm{C}$ & -1.95636700 & 0.69745100 & -0.00004300 \\
$\mathrm{C}$ & -3.20689900 & 1.38952000 & 0.10366500 \\
$\mathrm{C}$ & -2.90791700 & 2.75631700 & 0.03679000 \\
$\mathrm{C}$ & -1.51097600 & 2.89294200 & -0.10022600 \\
$\mathrm{~N}$ & -0.95750300 & 1.62525100 & -0.11135900 \\
$\mathrm{C}$ & 0.65745300 & 4.08694300 & -0.25434400 \\
$\mathrm{C}$ & 1.46905300 & 5.27491400 & -0.46585600 \\
$\mathrm{C}$ & 2.75983500 & 4.86603100 & -0.41237200 \\
$\mathrm{C}$ & 2.72995200 & 3.42392200 & -0.17399100 \\
$\mathrm{~N}$ & 1.46069500 & 2.97081400 & -0.10507800 \\
$\mathrm{C}$ & 3.92832100 & 2.66098600 & -0.01297300 \\
$\mathrm{C}$ & -0.75419900 & -4.08216800 & -0.19952300 \\
$\mathrm{C}$ & -0.74252700 & 4.08140000 & -0.21107300 \\
$\mathrm{C}$ & -4.47120000 & 0.69788900 & 0.20411500 \\
$\mathrm{C}$ & -4.47611900 & -0.68530500 & 0.17171400 \\
$\mathrm{C}$ & -1.30829600 & -6.36220000 & 0.71865900 \\
\hline $\mathrm{C}$ & -1.50014600 & -5.37148600 & -0.25769900 \\
$\mathrm{C}$ & -1.48492100 & 5.37240500 & -0.27623900 \\
$\mathrm{C}$ & -5.72012700 & -1.50079100 & 0.29074600 \\
$\mathrm{C}$ & -5.71340700 & 1.52035500 & 0.28657600 \\
$\mathrm{C}$ & -2.41753100 & -5.63331800 & -1.28823400 \\
$\mathrm{C}$ & 5.18487300 & -3.48868800 & 0.10432900 \\
$\mathrm{C}$ & 5.19661800 & 3.47239500 & 0.05242100 \\
$\mathrm{C}$ & 5.50673500 & -4.20279600 & 1.26719500 \\
$\mathrm{C}$ & 6.67439700 & -4.96095700 & 1.33977600 \\
$\mathrm{C}$ & 7.55655300 & -5.04045900 & 0.25394300 \\
$\mathrm{C}$ & 7.23230800 & -4.32590500 & -0.90604000 \\
$\mathrm{C}$ & 6.06804300 & -3.56145000 & -0.98133000 \\
$\mathrm{C}$ & 6.07406400 & 3.52920000 & -1.03970800 \\
$\mathrm{C}$ & 7.23674800 & 4.29644900 & -0.98330700 \\
$\mathrm{C}$ & 7.56878600 & 5.02643200 & 0.16570300 \\
$\mathrm{C}$ & 6.68998000 & 4.96796600 & 1.25465200 \\
$\mathrm{C}$ & 5.52268700 & 4.20650900 & 1.20077200 \\
$\mathrm{C}$ & -2.00802000 & -7.86564600 & 0.66407600 \\
$\mathrm{C}$ & & & \\
$\mathrm{C}$ & & -6.84114200 & -1.33996200 \\
$\mathrm{C}$ & & &
\end{tabular}

\begin{tabular}{|c|c|c|c|}
\hline Atoms & $\mathrm{X}$ & $Y$ & Z \\
\hline $\mathrm{C}$ & -1.27575000 & 6.37501800 & 0.68429600 \\
\hline C & -1.97637500 & 7.57798300 & 0.62747600 \\
\hline C & -2.90631900 & 7.82977500 & -0.38936200 \\
\hline C & -3.11603500 & 6.82774400 & -1.34573100 \\
\hline C & -2.42260900 & 5.62076000 & -1.29200100 \\
\hline C & -6.72645200 & 1.40220500 & -0.67812500 \\
\hline C & -7.87324700 & 2.19158700 & -0.61026700 \\
\hline C & -8.03075200 & 3.11704100 & 0.42292800 \\
\hline C & -7.03108700 & 3.24703700 & 1.38787400 \\
\hline C & -5.88222000 & 2.45862700 & 1.31835900 \\
\hline C & -6.07483500 & -2.41550400 & -0.71448100 \\
\hline C & -7.22770700 & -3.19267700 & -0.60072800 \\
\hline C & -8.04497500 & -3.07476000 & 0.52419100 \\
\hline C & -7.70087600 & -2.17312000 & 1.53287000 \\
\hline C & -6.55045900 & -1.39448400 & 1.41744700 \\
\hline C & 8.80292100 & -5.89068500 & 0.32501300 \\
\hline C & 8.84556200 & 5.83038900 & 0.23477000 \\
\hline C & -3.70226200 & -9.12084800 & -0.40226600 \\
\hline C & -3.63970200 & 9.14737000 & -0.46714200 \\
\hline $\mathrm{H}$ & -8.92473400 & 3.73169600 & 0.47533600 \\
\hline $\mathrm{H}$ & -7.14474200 & 3.96145000 & 2.19836200 \\
\hline $\mathrm{H}$ & -8.64402600 & 2.08542800 & -1.36839100 \\
\hline $\mathrm{H}$ & -6.60882900 & 0.68659000 & -1.48529100 \\
\hline $\mathrm{H}$ & -5.10986600 & 2.56044600 & 2.07502100 \\
\hline $\mathrm{H}$ & -8.32832300 & -2.07667900 & 2.41435900 \\
\hline $\mathrm{H}$ & -6.28699100 & -0.69613700 & 2.20512500 \\
\hline $\mathrm{H}$ & -8.94190100 & -3.68059600 & 0.61466600 \\
\hline $\mathrm{H}$ & -7.48814800 & -3.88846900 & -1.39341700 \\
\hline $\mathrm{H}$ & -5.44618000 & -2.50634900 & -1.59539900 \\
\hline $\mathrm{H}$ & -3.60925400 & 3.57360200 & 0.08115800 \\
\hline $\mathrm{H}$ & -3.62030700 & -3.56661800 & 0.06873800 \\
\hline $\mathrm{H}$ & -2.57158000 & -4.88956200 & -2.06386100 \\
\hline $\mathrm{H}$ & -3.80324500 & -7.02246000 & -2.15755800 \\
\hline $\mathrm{H}$ & -1.84252100 & -8.31263100 & 1.43660500 \\
\hline $\mathrm{H}$ & -0.61136200 & -6.17830900 & 1.53022900 \\
\hline $\mathrm{H}$ & -4.63813900 & -9.03615700 & 0.16432500 \\
\hline $\mathrm{H}$ & -3.13232200 & -9.94568900 & 0.03575200 \\
\hline $\mathrm{H}$ & 9.57093200 & -5.53447500 & -0.36769500 \\
\hline $\mathrm{H}$ & 9.22983500 & -5.89249300 & 1.33265100 \\
\hline $\mathrm{H}$ & 6.90511400 & -5.49846900 & 2.25643900 \\
\hline $\mathrm{H}$ & 4.83740500 & -4.15658200 & 2.12125300 \\
\hline $\mathrm{H}$ & 5.83807600 & -3.01396400 & -1.89076100 \\
\hline $\mathrm{H}$ & 7.89980600 & -4.36713900 & -1.76336800 \\
\hline $\mathrm{H}$ & 1.08065800 & -6.28331500 & -0.58690500 \\
\hline $\mathrm{H}$ & 3.63428100 & -5.48511300 & -0.46307900 \\
\hline $\mathrm{H}$ & 6.23380900 & -1.28321900 & 0.55649500 \\
\hline $\mathrm{H}$ & 6.23805100 & 1.27068900 & 0.53781500 \\
\hline $\mathrm{H}$ & 5.83549300 & 2.97331600 & -1.94180100 \\
\hline $\mathrm{H}$ & 4.85343400 & 4.18074000 & 2.05572900 \\
\hline $\mathrm{H}$ & 6.92040500 & 5.52771800 & 2.15788300 \\
\hline $\mathrm{H}$ & 7.89532700 & 4.33125100 & -1.84792000 \\
\hline
\end{tabular}




\begin{tabular}{cccc}
\hline Atoms & $\mathrm{X}$ & $\mathrm{Y}$ & $\mathrm{Z}$ \\
\hline $\mathrm{H}$ & 9.69322700 & 5.20415100 & 0.54024700 \\
$\mathrm{H}$ & 8.76621700 & 6.64595000 & 0.95941400 \\
$\mathrm{H}$ & 3.64775700 & 5.46688700 & -0.53685400 \\
$\mathrm{H}$ & 1.09568700 & 6.27169000 & -0.64753000 \\
$\mathrm{H}$ & -2.59900100 & 4.86369300 & -2.04963900 \\
$\mathrm{H}$ & -0.57001800 & 6.19797800 & 1.48979900 \\
$\mathrm{H}$ & -1.80199800 & 8.33153600 & 1.39160900 \\
$\mathrm{H}$ & -3.83158600 & 6.99442500 & -2.14708800 \\
$\mathrm{H}$ & -3.77683100 & 9.58853200 & 0.52456200 \\
\hline
\end{tabular}

\begin{tabular}{crrr}
\hline Atoms & $\mathrm{X}$ & $\mathrm{Y}$ & $\mathrm{Z}$ \\
\hline $\mathrm{H}$ & 0.05165600 & -1.51613700 & -0.12417600 \\
$\mathrm{H}$ & 0.05737800 & 1.51310000 & -0.13004000 \\
$\mathrm{H}$ & 8.58692800 & -6.93416900 & 0.06340100 \\
$\mathrm{H}$ & 9.09880900 & 6.26415700 & -0.73749100 \\
$\mathrm{H}$ & -3.96748300 & -9.39800100 & -1.42682200 \\
$\mathrm{H}$ & -3.08408300 & 9.87476500 & -1.07234700 \\
$\mathrm{H}$ & -4.62602100 & 9.03042200 & -0.92586900 \\
$\mathrm{~S}$ & 2.85973500 & -0.00516700 & -0.07277100 \\
\hline
\end{tabular}

